\title{
Asymptotics of quantum channels: conserved quantities, an adiabatic limit, and matrix product states
}

\author{
Victor V. Albert ${ }^{1,2}$ \\ ${ }^{1}$ Walter Burke Institute for Theoretical Physics and Institute for Quantum Information and Matter, California Institute of Technology, \\ Pasadena, California, USA \\ ${ }^{2}$ Yale Quantum Institute, Departments of Applied Physics and Physics, Yale University, New Haven, Connecticut, USA
}

This work derives an analytical formula for the asymptotic state-the quantum state resulting from an infinite number of applications of a general quantum channel on some initial state. For channels admitting multiple fixed or rotating points, conserved quantities - the left fixed/rotating points of the channeldetermine the dependence of the asymptotic state on the initial state. The formula stems from a Noether-like theorem stating that, for any channel admitting a full-rank fixed point, conserved quantities commute with that channel's Kraus operators up to a phase. The formula is applied to adiabatic transport of the fixed-point space of channels, revealing cases where the dissipative/spectral gap can close during any segment of the adiabatic path. The formula is also applied to calculate expectation values of noninjective matrix product states (MPS) in the thermodynamic limit, revealing that those expectation values can also be calculated using an MPS with reduced bond dimension and a modified boundary.

\section{Introduction \& outline}

A quantum channel $\mathcal{A}$ (also called a quantum markov chain, Kraus map, or completely-positive tracepreserving map) is the most general map between two quantum systems. Channels enjoy a range of applications, primarily in the quantum information community [1], but also in studies of matrix product states [2, 3], entanglement renormalization [4, 5], computability theory [6], and even biological inference processes [7]. Whenever one studies such maps, a key question to ask is:

What information from an initial state $\rho$ survives under repeated application of $\mathcal{A}$ ?

Answering this question helps determine how to properly initialize a quantum computer [8, 9], optimize the preparation of certain exotic states [10, 11] (part of the active field of reservoir engineering [12]), and even determine properties of matrix product states in the thermodynamic limit [3]. Quantum channels have already been engineered in, e.g., trapped ion [13] and IBM's circuit QED [14] platforms, and reliable engineering of arbitrary channels is actively being investigated [15-17]. Thus, it is important for the quantum community to be on firm ground with respect to question (1).

There have been several studies tackling question (1), but many of them focused on either (A) Lindbladian channels, (B) on channels containing only fixed points (eigenmatrices with eigenvalue +1 ) and no rotating points (eigenmatrices with eigenvalue having modulus +1 ), and/or (C) channels admitting a fullrank steady state (i.e., channels that do not cause an entire subspace to decay to zero upon infinite applications). An answer to question (1) for the most general case is different than for any one of the specific cases (A-C). For example, due to the ability of general channels to permute states upon only one application, an answer [8] for channels generated by a Lindbladian [18-21] does not cover the general case. To my knowledge, the following closely related works considered channels with rotating points in detail. Reference [22] (supplemented with a guided tour by Wolf [23]) and an appendix of Ref. [24] derive a limit of infinite applications of a general channel, carefully canceling perpetually oscillating phases. There have been two different decompositions associated with general channels: [23, Thm. 8] being a fine-grained blockdecomposition of the asymptotic subspace - the subspace that survives under repeated application of the channel-and [25, Thm. 2] being a coarser algebraic decomposition of the Kraus operators into blocks of noiseless subsystems (see Sec. 5.2 for details). The works [26-28] as well as older literature (e.g., [2, 29]; see also [23]) consider classes of channels with rotating points, but such channels do not admit a decaying subspace. In fact, none of the works listed fully address conserved quantities of channels admitting decay.

In the MPS context [3], a state's transfer channel $\mathcal{A}$ (also called a double tensor [30]) is a channel whose Kraus operators are used to construct the MPS. The limit of infinite applications of $\mathcal{A}$ is intimately related to the thermodynamic limit of the MPS. Question (1) 
is then equivalent to

\section{What part of the MPS and its boundary conditions survive in the thermodynamic limit?}

The exact connection between quantum channels and MPSs has been well-studied for the case when the MPS is injective - when its transfer channel has only one fixed point and no rotating points. However, noninjective MPS (with $\mathcal{A}$ having multiple fixed/rotating points) arise naturally in physical systems, ranging from anti-ferromagnets and Majumdar-Ghosh states [3] to more general spin chains [2, 31, 32] and systems with topological degeneracy [33, 34]. Non-injectivity is also ever-present in fermionic MPS [35] due to fermion parity preservation. In such MPS, effects due to the interaction of "twisted" boundary conditions with decaying bond degrees of freedom can persist in the thermodynamic limit, thus needing to be taken into account. Applying results about $\mathcal{A}$, this work derives a consistent thermodynamic limit, improving on Ref. [36], Eqs. (130-133) (cf. [37, 38]). I also provide general results about expectation values, answering question (2) and showing how to absorb any "twisted" boundary effects into the boundary matrix of an MPS with smaller bond dimension.

The first part of this manuscript answers question (1) in a manner meant to be accessible to a large part of the quantum community. Section 2 introduces some notation and the general asymptotic framework. In Sec. 3, I start with channels $\mathcal{E}$ that do not admit decay, i.e., have a full-rank fixed-point. In Sec. 4, I pad the Kraus operators of $\mathcal{E}$ to obtain the channel $\mathcal{A}$ admitting a decaying subspace. It is shown how to construct the conserved quantities of $\mathcal{A}$ from those of $\mathcal{E}$. In Sec. 5, I work in known results about the finer algebraic block decomposition of the asymptotic subspace, addressing question (1) for various subspaces. Special care is taken regarding asymptotics of irreducible channels - channels with a unique fixed point and one or more rotating points - as those differ substantially from any other case.

The second part of this work puts the results from the first part to use in two applications. Given a general channel $\mathcal{A}$, it is shown that its restriction $\mathcal{E}$ to the maximal invariant subspace is sufficient for both applications. Section 6 develops an adiabatic limit for fixed-point spaces of continuous families of channels $\mathcal{A}^{(s)}$, showing that this limit quickly reduces to the limit of the corresponding Lindbladian channels $\mathcal{E}^{(s)}-\mathcal{I}$. This answers the third question posed in this work,

What are any differences between the fixed-point adiabatic limits of Hamiltonians and channels?

It is shown that a gap condition need only hold for a part of the channel, and the part acting on the decaying subspace can have its gap close without affecting the limit. Section 7 applies to matrix product states, where it is shown how one can calculate expectation values in the thermodynamic limit of MPS associated with $\mathcal{A}$ using MPS associated with $\mathcal{E}$.

\section{Asymptotics of channels}

The canonical form of a quantum channel $\mathcal{A}$ and its adjoint $\mathcal{A}^{\ddagger}$ (a generalization of the Heisenberg picture defined under the Frobenius norm) is [39-41]

$$
\mathcal{A}(\rho)=\sum_{\ell} A^{\ell} \rho A^{\ell \dagger} \quad \text { and } \quad \mathcal{A}^{\ddagger}(O)=\sum_{\ell} A^{\ell \dagger} O A^{\ell} \text {, }
$$

where $\mathcal{A}$ acts on states $\rho$ and $\mathcal{A}^{\ddagger}$ on operators $O$. The matrices $A^{\ell}$ are called the Kraus operators of $\mathcal{A} \equiv$ $\left\{A^{\ell}\right\}$, eq. (4) is the Kraus form of $\mathcal{A}$, and the only requirement for the channel to be trace preserving is (for $I$ identity)

$$
\mathcal{A}^{\ddagger}(I)=\sum_{\ell} A^{\ell \dagger} A^{\ell}=I .
$$

The Kraus operators are assumed to be $D$ dimensional here, but the intuition presented here can be extended to certain infinite-dimensional cases [42]. Such channels can be represented as matrices acting on a vectorized density matrix, i.e., the $D \times D$ matrix $\rho$ written as a $D^{2}$-dimensional vector. Vectorization essentially "flips" the bra part in each of the outer products making up $\rho$ and $\mathcal{A}$ is written as a $D^{2} \times D^{2}$ matrix of the form $\hat{\mathcal{A}}=\sum_{\ell} A^{\ell} \otimes \overline{A^{\ell}}$ acting on the vectorized $\rho$ strictly from the left (with $\bar{A}$ being the complex conjugated $A$ ). This matrix or Liouville representation of $\mathcal{A}$ [43] is equivalent to the Kraus representation (4), and I slightly abuse notation by ignoring hats and not distinguishing the two.

In the matrix representation, channels can be studied in terms of their eigenvalues and eigenmatrices. The eigenvalues of all channels are contained in the unit disk, and this work focuses on the eigenvalues/matrices $\Psi$ on the periphery of that disk, i.e.,

$$
\mathcal{A}(\Psi)=e^{i \Delta} \Psi \quad \text { for some real } \Delta .
$$

Such eigenmatrices are called the channel's (right) rotating points, and those with $\Delta=0$ are called fixed points. The rotating points do not have to be physical states themselves; e.g., $\Psi_{k k^{\prime}}=|k\rangle\left\langle k^{\prime}\right|$ is a rotating point of the channel $\mathcal{A}(\cdot)=U(\cdot) U^{\dagger}$, where $|k\rangle,\left|k^{\prime}\right\rangle$ are eigenstates of $U$. Since $\mathcal{A}$ may not be normal $\left(\left[\mathcal{A}, \mathcal{A}^{\ddagger}\right] \neq 0\right)$, the eigenmatrices $J$ of its adjoint left rotating points - may be different from $\Psi$ :

$$
\mathcal{A}^{\ddagger}(J)=e^{-i \Delta} J \text {. }
$$

Left rotating points will be called conserved quantities because their expectation value is either constant or oscillates with successive powers of $\mathcal{A}$, but does not decay:

$$
\operatorname{Tr}\left\{J \mathcal{A}^{n}(\rho)\right\}=\operatorname{Tr}\left\{\mathcal{A}^{\ddagger n}(J) \rho\right\}=e^{-i n \Delta} \operatorname{Tr}\{J \rho\} .
$$


The general block structure of $\Psi$ 's is already wellknown [24, 44-47], and here the focus is on the structure of the J's. It is important to note that there are as many conserved quantities as there are rotating points (more technically, the Jordan normal form of $\mathcal{A}$ contains only trivial Jordan blocks for all eigenvalues on the periphery of the unit disk; see, e.g., Prop 6.2 in Ref. [23]). All channels have at least one fixed point [48] with corresponding conserved quantity being the identity, always conserved due to eq. (5).

In the limit of many applications of $\mathcal{A}$, all eigenmatrices with eigenvalues not on the periphery of the unit disk will become irrelevant and all that will be left of the channel is the projection onto the subspace spanned by the rotating points. The collective effect of many applications of $\mathcal{A}$ is quantified by the channel's asymptotic projection $\mathcal{P}_{\mathcal{A}}$,

$$
\mathcal{P}_{\mathcal{A}}(\cdot) \equiv \lim _{m \rightarrow \infty} \mathcal{A}^{\alpha_{m}}(\cdot),
$$

which projects onto the eigenspace of the peripheral spectrum of the channel. The extra parameter $\alpha$ allows one to take the limit in such a way as to remove the eigenvalues $e^{i \Delta}$ arising from application of $\mathcal{A}$ on $\rho$. For any $\Delta=\frac{2 \pi}{N} n$ (for some positive integers $n, N)$, rotating points of $\mathcal{A}$ are fixed points of $\mathcal{A}^{N}$, so one simply takes $\alpha_{m}=N m$ to get rid of the extra phases. In this context, one can think of $\mathcal{A}$ as being an "asymptotic" root of $\mathcal{P}_{\mathcal{A}}$ [49]. Other $\Delta$ which are not rational multiples of $2 \pi$ can similarly be removed to arbitrary accuracy [22-24] by remembering that irrational numbers are limits of sequences of rationals. The above limit is a direct generalization of the large time limit of Lindbladian channels $\mathcal{A}_{t}=e^{t \mathcal{L}}$ for some Lindbladian $\mathcal{L}$. However, in that case, $\lim _{t \rightarrow \infty} e^{t \mathcal{L}}$ produces residual unitary evolution which is not so easily removed by clever manipulation of the limit.

The asymptotic projection is expressible in terms of (superoperator) projections onto the eigenspaces of the rotating points,

$$
\mathcal{P}_{\mathcal{A}}(\rho)=\sum_{\Delta, \mu} \Psi_{\Delta \mu} \operatorname{Tr}\left\{J^{\Delta \mu \dagger}(\rho)\right\}
$$

where the rotating points are indexed by their eigenvalue $e^{i \Delta}$ and $\mu$ counts any degeneracies for each $\Delta$. In that sense, conserved quantities are as important as fixed points despite being less well-understood. Conveniently, the rotating points and their corresponding conserved quantities can be made biorthogonal, $\operatorname{Tr}\left\{J^{\Delta \mu \dagger} \Psi_{\Theta \nu}\right\}=\delta_{\Delta \Theta} \delta_{\mu \nu}$. The $\Psi$ 's thus determine the basis elements of a generalized Bloch vector [50, 51] of the asymptotic state $\mathcal{P}_{\mathcal{A}}(\rho)$ while the $J$ 's determine the coefficients of said Bloch vector. The biorthogonality condition easily implies that $\mathcal{P}_{\mathcal{A}}$ is really a projection $-\mathcal{P}_{\mathcal{A}}^{2}=\mathcal{P}_{\mathcal{A}}$.

The asymptotic projection for a channel with unique fixed point acts as $\mathcal{P}_{\mathcal{A}}(\rho)=\Psi \operatorname{Tr}\{\rho\}=\Psi$.

\begin{tabular}{|c|c|c|c|}
\hline & $\begin{array}{c}\text { Fixed point } \\
\text { unique? }\end{array}$ & $\begin{array}{c}\exists \text { full-rank } \\
\text { fixed point? }\end{array}$ & $\begin{array}{l}\exists \text { rotating } \\
\text { point? }\end{array}$ \\
\hline ergodic $[53-56]$ & Yes & & \\
\hline faithful [here] & & Yes & \\
\hline irreducible $[23,57]$ & Yes & Yes & \\
\hline mixing $[56]$ & Yes & & No \\
\hline primitive $[22,58]$ & Yes & Yes & No \\
\hline
\end{tabular}
Channels with more non-trivial $\mathcal{P}_{\mathcal{A}}$ are therefore those
Table 1: Various types of channels. A blank entry means there is no requirement for that definition. For Lindbladians, mixing is also known as relaxing [27] and faithful is also known as minimal [59]. Primitive is equivalent to strongly irreducible [58] and irreducible and aperiodic [60].

with multiple fixed or rotating points. As a simple example of such a channel, consider $\mathcal{A}=\{A\}$ acting on $2 \times 2$ matrices with one Kraus operator $A=\operatorname{diag}\left\{1, e^{i \theta}\right\}$. Such a channel sports two fixed points, the identity and the Pauli matrix $Z$, and two rotating points $\sigma_{ \pm}$with eigenvalues $\Delta= \pm \theta$. In fact, since there is only one Kraus operator, such a channel is actually unitary. For a non-unitary example, set $\theta=\pi$ (so $A=Z$ ) and add the Pauli matrix $X$ as another Kraus operator [normalizing both $A$ 's by $\frac{1}{\sqrt{2}}$ to satisfy trace preservation (5)]. This channel has the identity as the unique fixed point and $Y$ as the only rotating point with $\Delta=\pi$. Since both Kraus operators are Hermitian, the left and right fixed points are the same; we will see examples when they are not later. Other examples of $\mathcal{P}_{\mathcal{A}}$ come from recovery maps in quantum error-correction, which take a state which has undergone an error and project it back into the protected subspace of the quantum code [52].

\section{Faithful channels}

This part focuses on channels that do not contain a decaying subspace. This means that no populations $|\psi\rangle\langle\psi|$ decay completely to zero under many applications of the channel: $\left\langle\psi\left|\mathcal{P}_{\mathcal{E}}(|\psi\rangle\langle\psi|)\right| \psi\right\rangle \neq 0$ for all states $|\psi\rangle$, a channel $\mathcal{E}$, and its asymptotic projection $\mathcal{P}_{\mathcal{E}}$. Equivalently, the channel has to have one fixed point $\rho$ which is of full $\operatorname{rank}(\langle\psi|\rho| \psi\rangle>0$ for all $|\psi\rangle)$. The structural differences between such channels and channels which do admit decay warrant a special definition:

Definition. A channel $\mathcal{E} \equiv\left\{E_{\ell}\right\}$ is faithful if it admits a full-rank (i.e., faithful) fixed point $\rho$. In other words,

$$
\exists \rho>0 \text { such that } \mathcal{E}(\rho)=\rho .
$$

Here, I always use $\mathcal{E}$ to denote faithful channels and later show how $\mathcal{E}$ can be extended to channels $\mathcal{A}$ which act on a larger Hilbert space and admit a decaying subspace. In this sense, $\mathcal{E}$ is the faithful channel of $\mathcal{A}$. Note that the number of fixed points is independent of this condition, and Table 1 relates this definition to others. 
The first statement is regarding the relationship between the conserved quantities $J$ and the Kraus of operators of $\mathcal{E}$. It is a generalization of a theorem for fixed points of faithful channels [24, 61-64], which states that a conserved quantity $J$ with eigenvalue $\Delta=0$ commutes with all of the Kraus operators. It is shown that conserved quantities with $\Delta \neq 0$ commute up to a phase. For the aforementioned example $\mathcal{E}=\{E\}$ with $E=\operatorname{diag}\left\{1, e^{i \theta}\right\}$, the conserved quantity $\sigma_{+}$satisfies $\sigma_{+} E=e^{-i \theta} E \sigma_{+}$. This turns out to be true for all faithful channels. We state the result below, relegating all proofs for the appendix.

Proposition 1. Let $\mathcal{E}=\left\{E_{\ell}\right\}$ be a faithful channel. Let $J$ be a conserved quantity of $\mathcal{E}$, i.e.,

$$
\mathcal{E}^{\ddagger}(J)=e^{-i \Delta} J
$$

for some real $\Delta$. Then, for all $\ell$,

$$
J E_{\ell}=e^{-i \Delta} E_{\ell} J
$$

Next, I show that this formula imposes some restrictions on the peripheral spectrum of $\mathcal{E}$, and that unitary $J$ can be thought of as symmetries in a limited extension of Noether's theorem.

\subsection{Spectral restrictions}

Assuming $\mathcal{E}^{\ddagger}\left(J_{1}\right)=e^{-i \Delta_{1}} J_{1}$ and $\mathcal{E}^{\ddagger}\left(J_{2}\right)=e^{-i \Delta_{2}} J_{2}$, Eq. (13) implies that $\mathcal{E}^{\ddagger}\left(J_{1} J_{2}\right)=e^{-i\left(\Delta_{1}+\Delta_{2}\right)} J_{1} J_{2}$. Combined with the fact that there must be $\leq D^{2}$ conserved quantities, there are sometimes constraints on $\Delta$ such that there remain finitely many eigenvalues. Thus, I suggest that we divide conserved quantities into two types: nilpotent ones $J_{\text {nil }}$ and diagonalizable ones $J_{\mathrm{dgn}}$. Since $J_{\text {nil }}^{N}=0$ for $N \leq D$, the dimension of the Hilbert space, there are no restrictions on their eigenvalue. For a unitary channel $\mathcal{E}=\{U\}$, such conserved quantities are the coherences $|k\rangle\left\langle k^{\prime}\right|$ between eigenstates $|k\rangle \neq\left|k^{\prime}\right\rangle$ of $U$. On the other hand, projections $|k\rangle\langle k|$ onto eigenstates of $U$ are examples of $J_{\text {dgn }}$ with $\Delta=0$. While this is the only possible value for $J_{\mathrm{dgn}}$ of unitary channels, general channels admit special $J_{\text {dgn }}$ with $\Delta \neq 0$. For example, the channel $\mathcal{E}=\{X / \sqrt{2}, Z / \sqrt{2}\}$ admits conserved quantities $J_{\mathrm{dgn}} \in\{I, Y\}$ with eigenvalues \pm 1 , respectively. More generally, it turns out these special $J_{\text {dgn }}$ can have only $N$ th root-of-unity eigenvalues, with $N$ tightly bounded by the dimension of the range of $J_{\mathrm{dgn}}$.

Proposition 2. Let $\mathcal{E}=\left\{E_{\ell}\right\}$ be a faithful channel. Let $J_{\mathrm{dgn}}$ be such that $\mathcal{E}^{\ddagger}\left(J_{\mathrm{dgn}}\right)=e^{-i \Delta} J_{\mathrm{dgn}}$ for some real $\Delta$ and assume $J_{\mathrm{dgn}}$ is diagonalizable. Then, there exists an integer $n$ such that

$$
\Delta=\frac{2 \pi}{N} n \quad \text { for some } \quad N \leq\left\|J_{\mathrm{dgn}}\right\|_{1},
$$

where \|\|$_{1}$ is the trace norm.
Splitting the set of $J_{\mathrm{dgn}}$ into the ordinary $\Delta=0$ case and the above special case, one can classify conserved quantities and their corresponding rotating points (it is implied that $\Delta \neq 0$ in the latter two types):

I Ordinary conserved quantities: $\Delta=0$

II Nilpotent conserved quantities: $\Delta \in \mathbb{R}$

III Diagonalizable conserved quantities: $\Delta=\frac{2 \pi}{N} n$.

Types I and II exist for Lindbladian evolutions, but type III is unique to general channels. Type III quantities affect the block-decomposition of rotating points into noiseless factors; this is addressed in Sec. 5.

\subsection{Limited Noether-type theorem}

Let us assume a unitary conserved quantity, $J^{\dagger} J=$ $J J^{\dagger}=I$, and show that the above two propositions extend known results ([23], Prop. 6.7) from irreducible to faithful channels. Proposition 1 readily implies that $\mathcal{E}$ is covariant (more specifically, invariant or symmetric) under $J$,

$$
J \mathcal{E}(\rho) J^{\dagger}=\mathcal{E}\left(J \rho J^{\dagger}\right) \quad \forall \rho,
$$

so conserved quantities are symmetries of the channel. Such quantities are also important in determining how the set of channels decomposes into path-connected subsets [65, Thm. 5] (see also [66]). Proposition 2 implies that $J^{N \leq D}=I$, so the set $\left\{J^{n}\right\}_{n=0}^{N-1}$ forms the symmetry group $\mathbb{Z}_{N}$. Note that the symmetry group is never infinite for finite dimension $D$. Generalizing this, the set of unitary conserved quantities thus forms a finite group under which $\mathcal{E}$ is covariant. This is a one-way Noether-type theorem linking conserved quantities to symmetries (see Refs. [8, 67] or Ref. [68], Ch. 2.6, for the Lindbladian analogue). Note that this generalization is in a different direction from Ref. [69], which introduces measures quantifying the extent to which a quantum state breaks a symmetry. This one-way theorem cannot be extended to a two-way theorem because symmetries of a channel are not always conserved quantities. A simple counterexample is the channel $\mathcal{E}=\{X / \sqrt{2}, Z / \sqrt{2}\}$, for which the Hadamard operation $H$ taking $X \leftrightarrow Z$ is a symmetry, but is not conserved $\left[\mathcal{E}^{\ddagger}(H)=0\right]$.

\section{Extending to general channels}

Now let us extend faithful channels to channels which do not contain a full-rank fixed point. While Props. 1-2 break down for general channels, the extension below implies that, for every general channel, there is a corresponding faithful channel for which they hold.

Any faithful channel $\mathcal{E}=\left\{E_{\ell}\right\}$ can be extended to a channel $\mathcal{A}=\left\{A^{\ell}\right\}$ which contains a decaying 
subspace (also, transient subspace [70]). Specifically, the Kraus operators of $\mathcal{A}$ are

$$
A^{\ell}=\left(\begin{array}{cc}
E_{\ell} & A_{\mathbb{\boxplus}}^{\ell} \\
0 & A_{\mathbb{\Xi}}^{\ell}
\end{array}\right) \equiv\left(\begin{array}{cc}
A_{\mathbb{\boxplus}}^{\ell} & A_{\mathbb{\Xi}}^{\ell} \\
0 & A_{\mathbb{\Xi}}^{\ell}
\end{array}\right) .
$$

The zero is necessary by assumption: for $\mathcal{E}$ to act on the largest invariant subspace, $\mathcal{A}$ cannot take states out of that subspace. The dimensions of the square matrices $E_{\ell}$ and $A_{\mathbb{Q}}^{\ell}$ can differ, and the bounds of $\ell$ can change by padding the same $E$ with two different pairs of matrices in $\boxplus$ ("upper right") and $\square$ ("lower right") to make two different $A$ 's. The zero matrix in $\boldsymbol{\Psi}$ is necessary to make sure that $\boldsymbol{\Psi}$ is the largest invariant subspace; thus, all rotating points of $\mathcal{A}$ are the same as those of $\mathcal{E}$. In addition, $\mathcal{A}$ needs to be a legitimate channel, i.e., satisfy eq. (5). Writing out the $A^{\ell}$ 's in blocks [as in eq. (16)] yields the conditions

$$
\begin{aligned}
\sum_{\ell} A_{\boxplus}^{\ell \dagger} A_{\boxplus}^{\ell} & =P \\
\sum_{\ell} A_{\boxplus}^{\ell \dagger} A_{\boxplus}^{\ell} & =0 \\
\sum_{\ell}\left(A_{\boxplus}^{\ell}\right)^{\dagger} A_{\boxplus}^{\ell}+A_{\boxplus}^{\ell \dagger} A_{\boxplus}^{\ell} & =Q,
\end{aligned}
$$

where $Q$ is the projection on $\square$ and $P=I-Q$ is the projection onto $\boldsymbol{\Xi}$ (with $\operatorname{Tr}\{P\} \equiv D$ ). For each faithful channel $\mathcal{E}$, there are an infinite number of possible extensions $\mathcal{A}$. Conversely, an arbitrary channel $\mathcal{A}$ either is a faithful channel or contains one, i.e., once the largest invariant subspace $\boldsymbol{\Theta}$ is determined (via, say, an algorithm [71]), one will obtain the block decomposition (16). The remaining two completely positive maps associated with this decomposition of $\mathcal{A},\left\{A_{\mathbb{⿴}}^{\ell}\right\}$ and $\left\{A_{\square}^{\ell}\right\}$, are both trace-decreasing.

Now let us develop the required notation. Just like $P$ and $Q$ split the Hilbert space into two parts, they can be used to split the space of operators on a Hilbert space into four "corners" $\{\boldsymbol{\Theta}, \boldsymbol{\Theta}, \boldsymbol{\Psi}, \boldsymbol{\boxplus}\}[59]$. Each of the four corners corresponds to its own superoperator projection. For example,

$$
\mathcal{P}_{\mathbb{\Psi}}(O) \equiv P O Q \equiv O_{\mathbb{\Psi}}
$$

for any operator $O$. The other three projections are defined accordingly. One can graphically determine which corner a product of operators belongs to by multiplying their blocks as matrices (e.g., $A_{\mathbb{\boxplus}} B_{\mathbb{\Psi}} \in \Xi$ ). Moreover, the four-corners projections add graphically $\left(\mathcal{P}_{\boldsymbol{\Psi}}+\mathcal{P}_{\boxplus} \equiv \mathcal{P}_{\mathbb{\boxplus}}\right)$ and are Hermitian $\left(\mathcal{P}_{\boxplus}^{\ddagger}=\mathcal{P}_{\boxplus}\right)$. Analogous to studying operators in terms of their matrix elements, one can study superoperators in terms of their four-corners decomposition. For example,

$$
\mathcal{P}_{\boldsymbol{\Xi}} \mathcal{A} \mathcal{P}_{\mathbb{\boxplus}}(\rho)=P \mathcal{A}(Q \rho Q) P=\sum_{\ell} A_{\boxplus}^{\ell} \rho_{\mathbb{\boxplus}}\left(A_{\boxplus}^{\ell}\right)^{\dagger}
$$

is the map $\left\{A_{\boxplus}^{\ell}\right\}$ which transfers $\rho_{\mathbb{\boxplus}}$ from $\boldsymbol{\Xi}$ to $\boldsymbol{\Xi}$. "Diagonal" elements are denoted as $\mathcal{A}_{\boxplus} \equiv \mathcal{P}_{\boxplus} \mathcal{A} \mathcal{P}_{\boxplus}$ for convenience, so the faithful channel $\mathcal{E} \equiv \mathcal{P}_{\boldsymbol{\Psi}} \mathcal{A} \mathcal{P}_{\boldsymbol{\Xi}}$ and similarly $\left\{A_{\boxplus}^{\ell}\right\} \equiv \mathcal{P}_{\boxplus} \mathcal{A} \mathcal{P}_{\boxplus}$.

With conditions (16) and (17), $\mathcal{A}$ contains a decaying subspace of dimension $\operatorname{Tr}\{Q\}$ and the same rotating points as $\mathcal{E}$. But what about the conserved quantities? Those are not the same because, by trace preservation, they need to make sure that all state populations (and sometimes some coherences) in $\square$ are transferred to $\boldsymbol{\Psi}$. For example, the identity is (always) a conserved quantity of $\mathcal{A}$, but the analogous conserved quantity of $\mathcal{E}$ is $P$. Denoting the conserved quantities of $\mathcal{E}$ as $J_{\boldsymbol{\varpi}}$, it will now be shown how to extend them to form $J$, the conserved quantities of $\mathcal{A}$. Having defined this notation, it is easy to write out the conserved quantities of the extended channel $\mathcal{A}$.

Proposition 3. The conserved quantities of $\mathcal{A}$ corresponding to eigenvalues $e^{i \Delta}$ are

$$
J=J_{\boldsymbol{\Theta}}+J_{\boxminus}=J_{\boldsymbol{\Theta}}+\mathcal{R}_{\boxplus}^{(\Delta) \ddagger} \mathcal{A}^{\ddagger}\left(J_{\boldsymbol{\Theta}}\right),
$$

where $J_{\boldsymbol{\Psi}}$ are the conserved quantities of $\mathcal{A}_{\boldsymbol{\Xi}}=\mathcal{E}$ and

$$
\mathcal{R}_{\boxplus}^{(\Delta)}=-\left(\mathcal{A}-e^{i \Delta}\right)_{\mathbb{\boxplus}}^{-1} .
$$

An important corollary of the above proposition is that $J_{\mathbf{D}}=0$. After plugging in this formula for $J$ into $\mathcal{P}_{\mathcal{A}}$ (10), this means that the asymptotic projection has only two pieces:

$$
\mathcal{P}_{\mathcal{A}}=\mathcal{P}_{\boldsymbol{\Phi}} \mathcal{P}_{\mathcal{A}} \mathcal{P}_{\boldsymbol{\Xi}} \equiv \mathcal{P}_{\mathcal{E}}+\mathcal{P}_{\mathcal{A}} \mathcal{P}_{\boldsymbol{\Theta}},
$$

where the faithful projection (for Lindbladians, minimal projection [59])

$$
\mathcal{P}_{\mathcal{E}}(\cdot) \equiv \mathcal{P}_{\mathcal{A}} \mathcal{P}_{\boldsymbol{\Psi}}(\cdot)=\sum_{\Delta, \mu} \Psi_{\Delta \mu} \operatorname{Tr}\left\{J_{\boldsymbol{\Theta}}^{\Delta \mu \dagger} \cdot\right\}
$$

is the asymptotic projection of the faithful channel $\mathcal{E}$. The piece $\mathcal{P}_{\mathcal{E}}$ is responsible for preserving parts of an initial state $\rho$ which is in $\square$ while the piece $\mathcal{P}_{\mathcal{A}} \mathcal{P}_{\mathbb{\Xi}}$ is a channel mapping states from $\boxplus$ onto the subspaces spanned by the rotating points of $\mathcal{A}$, all located in $\boldsymbol{\Psi}$. The key result here is that the rotation induced by $\Delta$, besides inducing phases on the rotating points, also contributes to the decay of information from $\boxplus$ into $\boldsymbol{\Psi}$. Namely, the inverse of the resolvent (21) modulates the decoherence induced during the decay in a way that depends on how close the eigenvalues of $\mathcal{A}_{\mathbb{\boxplus}}$ are to the phases $e^{i \Delta}$. The $\Delta=0$ case reduces to known results ([24], Lemma 5.8; [71], Prop. 7),

$$
\mathcal{P}_{\mathcal{A}} \mathcal{P}_{\boxplus}=\mathcal{P}_{\mathcal{E}} \mathcal{A}(\mathcal{I}-\mathcal{A})_{\boxplus}^{-1},
$$

where the resolvent can be thought of as the quantum version of the fundamental matrix from classical Markov chains [72]. These formulas also reduce to the Lindbladian result ([59], Prop. 3) if we let $\mathcal{A}=e^{\mathcal{L}} \rightarrow \mathcal{I}+\mathcal{L}$ for some Lindbladian $\mathcal{L}$ and 
$e^{-i \Delta} \rightarrow 1-i \Delta$. In the Lindblad case, some dependence on $\Delta$ can be canceled by properly tuning $\mathcal{L}_{\mathrm{⿴}}$ ([68], Sec. 3.2.3).

Directly applying Prop. 3 allows us to find the asymptotic [71] (also, reachability [70]) probabilities of a given initial state $\rho$ to reach a particular subspace of $\boldsymbol{\Psi}$. The new result here is determination of the coherences reached by $\rho$, assuming knowledge of the left $\left(J^{\Delta \mu}\right)$ and right $\left(\Psi_{\Delta \mu}\right)$ rotating points of $\mathcal{E}$. To determine the coefficient $c_{\Delta \mu}$ in the asymptotic state $\mathcal{P}_{\mathcal{A}}(\rho)=\sum_{\Delta \mu} c_{\Delta \mu} \Psi_{\Delta \mu}(10)$, instead of applying $\mathcal{A}$ a sufficiently large number of times to determine $\mathcal{P}_{\mathcal{A}}$, simply calculate

$c_{\Delta \mu}=\operatorname{Tr}\left\{J_{\boxplus}^{\Delta \mu \dagger} \rho_{\boxplus}\right\}+\sum_{\ell} \operatorname{Tr}\left\{A_{\boxplus}^{\ell \dagger} J_{\boxplus}^{\Delta \mu \dagger} A_{\boxplus}^{\ell} \mathcal{R}_{\boxplus}^{(\Delta)}\left(\rho_{\Xi}\right)\right\}$,

where I used eq. (19) and cyclic invariance.

\section{Adding fixed-point structure}

We have yet to consider the fine-grained structure of the asymptotic subspace. This section builds up the most general asymptotic subspace from its minimal ingredients: channels admitting decoherence-free subspaces and irreducible channels. The latter can have Type III (see Sec. 3.1) rotating points in the general case, making the usual noiseless subsystem decomposition significantly different from the Lindbladian or unitary cases. This section concludes with an algorithm that outputs a properly organized $\mathcal{P}_{\mathcal{A}}$ given a channel $\mathcal{A}$.

\subsection{Decoherence-free subspaces}

Let us assume that now all of $\Phi$ consists of rotat-

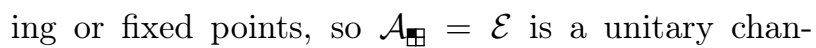
nel. An example of this case is $\mathcal{A}_{\boldsymbol{\Xi}}=\{E\}$, where $A_{\boldsymbol{\Xi}}=E=\operatorname{diag}\left\{1, e^{i \theta}\right\}$ is the Kraus operator that mentioned before. The necessary and sufficient condition on the $A$ 's (16) for this to hold is

$$
A_{\square}^{\ell}=a_{\ell} U
$$

for some unitary $U$, real $a_{\ell}$, and such that $\sum_{\ell}\left|a_{\ell}\right|^{2}=$ 1 to satisfy the condition (17a). Since there is no decay in $\Theta$, that portion forms a decoherence-free subspace (DFS) [73] and $\mathcal{P}_{\mathcal{E}}=\mathcal{P}_{\boldsymbol{\Psi}}$. The form of $A_{\varpi}$ also implies that $\mathcal{P}_{\boldsymbol{\Psi}} \mathcal{A} \mathcal{P}_{\boldsymbol{U}}=0$. The rotating points and conserved quantities of $\mathcal{E}$ - outer products of eigenstates of $U$-are of course the same.

Adding in decay, the form (16) of $A$ with the above restriction on $A_{\Xi}$ generalizes the previous DFS condition from eq. (11) of Ref. [74] (see also Refs. [75, 76] for different formulations). The difference is that now $A_{\boxplus}$ does not have to be zero, so information from $\boxminus$ flows into the DFS $\boxplus$. For example, in quantum errorcorrection, $\boxplus$ is the logical subspace, $\boxplus$ is the orthogonal error subspace, and the piece $\mathcal{P}_{\mathcal{A}} \mathcal{P}_{\boxplus}$ plays the role of a "recovery channel" which attempts to recover the leaked information after an error [52]. It turns out one can remove the inverse term from $\mathcal{P}_{\mathcal{A}} \mathcal{P}_{\mathrm{\Xi}}$, putting the piece in Kraus form. Setting $A_{\boxplus}=0$ and $A_{\boxplus}=P$ (unitary evolution within DFS is trivial) eliminates $\mathcal{A}_{\boxplus}$ and reduces $\mathcal{P}_{\mathcal{A}} \mathcal{P}_{\boxplus}$ to the transfer map (19)，

$$
\mathcal{P}_{\mathcal{A}} \mathcal{P}_{\boxplus}=\mathcal{P}_{\Xi} \mathcal{A} \mathcal{P}_{\boxplus}
$$

with Kraus operators $A_{\boxplus}$. Condition (17b) on $A_{\boxplus}$ reduces to $\sum_{\ell} A_{\boxplus}^{\ell}=0$, which is automatically satisfied by the set of operators $\left\{ \pm A_{\mathbb{E}}^{\ell} / \sqrt{2}\right\}$. However, the channel created by those operators is the same as $\left\{A_{\boxplus}^{\ell}\right\}$, so $\mathcal{P}_{\mathcal{A}}$ embeds an arbitrary recovery channel from the error subspace $\boxplus$ to code subspace $\boxplus$.

\subsection{Irreducible channels}

On the opposite side from DFS channels are the $i r$ reducible channels - channels admitting a unique fullrank fixed point. We find $\mathcal{P}_{\mathcal{A}}$ for such channels below, including the presence of any additional rotating points - a case unique to non-Lindbladian channels.

For Lindbladian channels, there is only one asymptotic state $(10) \mathcal{P}_{\mathcal{A}}(\rho)=\varrho$, the unique full-rank (on $\boxplus)$ fixed point of the channel. For general channels, this situation is complicated by any Type III rotating points. An example is the channel $\mathcal{E}=\mathcal{A}_{\boxplus}=$ $\{X / \sqrt{2}, Y / \sqrt{2}\}$, admitting two conserved quantities $\{I, Z\}$. Due to Prop. 2, all conserved quantities $J_{\boldsymbol{\Xi}}$ for irreducible channels are unitary (on $\boxplus$ ) and their corresponding rotating points are simply $\Psi=J_{\mathbf{⿴ 囗 十}} \varrho$. Moreover, conserved quantities are powers of a single unitary

$$
J_{\boldsymbol{\Theta}}=\sum_{n \in \mathbb{Z}_{N}} \omega^{n} \Pi_{n},
$$

where $\omega=e^{i \frac{2 \pi}{N}}$ is this quantity's eigenvalue (for some $N \leq D$, the total dimension) and $\Pi_{n}$ is the projection onto the eigenspace of eigenvalue $\omega^{n}[23$, Thm. 6.6; see also proof of Prop. 2]. We can thus represent them as $J_{\mathbb{\Theta}}^{\mu}$, where $\mu \in \mathbb{Z}_{N}$ is literally the $\mu$ th power of $J_{\boldsymbol{\Xi}}$. This $J_{\boldsymbol{\Xi}}$ must commute with $\varrho$; otherwise, $J_{\boldsymbol{⿴}} \varrho J_{\mathbf{\Theta}}^{\dagger}$ would be a different fixed point, violating irreducibility of $\mathcal{E}$.

Biorthogonality between the rotating points and conserved quantities with different eigenvalues and $\operatorname{Tr}\{\varrho\}=1$ imply that the following must be true:

$$
\operatorname{Tr}\left\{J_{\square}^{\mu \dagger} \Psi_{\nu}\right\}=\sum_{n \in \mathbb{Z}_{N}} \omega^{(\nu-\mu) n} \operatorname{Tr}\left\{\Pi_{n} \varrho\right\}=\delta_{\mu \nu} .
$$

Satisfaction of these $N$ equations forces $\operatorname{Tr}\left\{\Pi_{n} \varrho\right\}=$ $1 / N$. A striking fact is that the $\Psi$ 's themselves are no longer orthonormal under the Hilbert-Schmidt inner product, $\operatorname{Tr}\left\{\Psi_{\mu}^{\dagger} \Psi_{\nu}\right\} \not \subset \delta_{\mu \nu}$. This was the case for Lindbladian channels [59], and a simple counterexample ${ }^{1}$ shows this is not true generally.

$$
{ }^{1} \text { Let } \mathcal{E}=\left\{|2\rangle\langle 0|,| 2\rangle\left\langle 1\left|, \sqrt{\frac{2}{3}}\right| 1\right\rangle\left\langle 2\left|, \sqrt{\frac{1}{3}}\right| 0\right\rangle\langle 2|\right\} \text { act on }
$$


There is an alternative characterization [22, 29] of the subspace spanned by the rotating points, popularized by Wolf [23, Thm. 6.16]. Instead of using $\Psi_{\mu}$, one uses the basis

$$
\widetilde{\Psi}_{n} \equiv \Pi_{n} \varrho=\Pi_{n} \varrho \Pi_{n}
$$

of $\varrho$ projected onto eigenspaces of $J$. For our example $\mathcal{E}$, this basis is $\widetilde{\Psi}_{0}=|0\rangle\langle 0|$ and $\widetilde{\Psi}_{1}=|1\rangle\langle 1|$, and $\mathcal{E}$ permutes one with the other. This characterization allows one to decompose the space of rotating points into finer blocks (in the example, two 1-by-1 blocks). The $\widetilde{\Psi}_{n}$ are not eigenmatrices - they are permuted by $\mathcal{E}$ (see proof of Prop. 2)-but they are fixed points of $\mathcal{E}^{N}$. However, unlike $\Psi_{\mu}$, this basis is orthogonal.

As for the asymptotic state (10), despite there being only one fixed point, the rotating points also have to be included:

$$
\mathcal{P}_{\mathcal{E}}(\cdot)=\sum_{\mu \in \mathbb{Z}_{N}} \operatorname{Tr}\left\{J_{\mathbb{\Theta}}^{\mu \dagger}(\cdot)\right\} J_{\boxplus}^{\mu} \varrho=N \sum_{n \in \mathbb{Z}_{N}} \operatorname{Tr}\left\{\Pi_{n}(\cdot)\right\} \Pi_{n} \varrho,
$$

where I plugged in eq. (28) and simplified. Extending from $\mathcal{P}_{\mathcal{E}}$ to the full $\mathcal{P}_{\mathcal{A}}$ just substitutes $J_{\mathbb{R}}^{\mu}$ for the first $J_{\mathbb{\Theta}}^{\mu}$, with $J_{\mathbb{\boxplus}}^{\mu}$ determined by Prop. 3 .

\subsection{Noiseless subsystems}

The DFS case in Sec. 5.1 and the irreducible case in Sec. 5.2 are the two building blocks out of which one constructs the most general rotating point structures. Focusing on the $\boxminus$ space, a tensor product of a unitary channel and an irreducible channel $\left\{R_{\ell}\right\}$ has Kraus operators (16) [25]

$$
A_{\boxplus}^{\ell}=U \otimes R_{\ell}
$$

In Lindbladians, such a combination of a DFS and an auxiliary subspace with a unique fixed point is collectively called a noiseless subsystem (NS) [77]. Its asymptotic state would then be expressible in the matrix basis $|k\rangle\left\langle k^{\prime}\right| \otimes \varrho$, where $|k\rangle,\left|k^{\prime}\right\rangle$ are eigenstates of $U$ with eigenvalues $\lambda_{k}, \lambda_{k^{\prime}}$ and $\varrho$ is the unique fixed point of $\left\{R_{\ell}\right\}$ (e.g., [8]). However, the Type III rotating points of $\left\{R_{\ell}\right\}$ necessitate another index in the general case. Now, the rotating points are $\Psi_{k k^{\prime} \mu}=|k\rangle\left\langle k^{\prime}\right| \otimes J^{\mu} \varrho$, satisfying the eigenvalue equation

$$
\mathcal{A}\left(\Psi_{k k^{\prime} \mu}\right)=e^{i\left(\lambda_{k}-\lambda_{k^{\prime}}+\frac{2 \pi}{N} \mu\right)} \Psi_{k k^{\prime} \mu} .
$$

This makes contact with [22, Thm. 9], showing that the most general peripheral eigenvalues are combinations of eigenvalues of a unitary $U$ and a root of unity stemming from a Type III rotating point. It is not generally possible to make an orthonormal basis of

$\{|0\rangle,|1\rangle,|2\rangle\}$. This channel has a fixed point $\varrho=$ $\operatorname{diag}\{1 / 6,1 / 3,1 / 2\}$, and $J=\operatorname{diag}\{1,1,-1\}$. Eigenmatrices $\varrho$ and $J \varrho$ are not orthogonal. rotating points when the irreducible factor admits rotating points (see previous subsection).

Noiseless blocks can further be stacked to form the most general asymptotic subspace, corresponding to Kraus operators

$$
A_{\boxplus}^{\ell}=\bigoplus_{\varkappa} U_{\varkappa} \otimes R_{\ell, \varkappa},
$$

where $U_{\varkappa}$ is unitary and $\left\{R_{\ell, \varkappa}\right\}$ for each $\varkappa$ is irreducible [25]. This blocks-of-factors structure or shape of $A_{\mathbb{\Psi}}^{\ell}$ is the most general form of an informationpreserving structure [24]. Rotating points and conserved quantities of the form from the previous subsection can be constructed for each block to form a canonical basis (i.e., a basis respecting the block structure). It is well-known among experts (see, e.g., [78]) that the conserved quantities $\left\{J_{\boldsymbol{B}}\right\}$ form a $m a$ trix algebra - a vector space (where the vectors are matrices) that is closed under multiplication and the conjugate transpose operation. It is important to keep in mind that all of this extra structure in $\boxplus$ does not put any constraints on the remaining parts $\left\{A_{\boxplus}, A_{\boxplus}\right\}$ of $\mathcal{A}$, the extension of $\mathcal{E}$ (as long as eqs. (17) are satisfied). The extended conserved quantities $J$ do not have to form a matrix algebra.

\subsection{How to find $\mathcal{P}_{\mathcal{A}}$}

There exist several algorithms to determine the shape (34) of $\mathcal{A}[24,25,62,79-82]$. A straightforward way [24] to find the form (34) for a general channel $\mathcal{A}$ is to diagonalize $\mathcal{A}$ and apply standard matrix algebra techniques $[79,81]$ to find a canonical basis for the algebra of conserved quantities in $\boxplus$. Using Prop. 3, I slightly extend the algorithm from Ref. [24] to one that finds and organizes not just the conserved quantities restricted to $\boxplus$, but the full conserved quantities as well. Once again, the main new inclusion is the determination of conserved quantities whose eigenvalue is modulus one (as opposed to exactly one).

\section{Algorithm. Finding and organizing $\mathcal{P}_{\mathcal{A}}$}

Find the rotating points $\Psi$ and conserved quantities $J$ by diagonalizing $\mathcal{A}$

Construct $\mathcal{P}_{\mathcal{A}}$ and $P$, the projection onto range $\left\{\mathcal{P}_{\mathcal{A}}(I)\right\}$

Find the projected conserved quantities $J_{\mathbf{⿴ 囗 十}} \equiv P J P$

Decompose the algebra spanned by $J_{\boldsymbol{⿴}}$ into canonical form using, e.g., Refs. [79, 81]

Determine a canonical basis $\Psi$ for the rotating points and $J_{\boxplus}$ for the conserved quantities

Extend $J_{\boldsymbol{G}}$ to $J$ via Prop. 3.

Note that $\boxplus$ is the range of $\mathcal{P}_{\mathcal{A}}(I)$, i.e., $\mathcal{P}_{\mathcal{A}}(I) \propto$ $P$, because $I$ is dual to the maximally mixed fixed point $\frac{1}{\operatorname{Tr}\{P\}} P$ and is the only conserved quantity with nonzero trace. 


\section{Application: Adiabaticity}

In this section, an adiabatic limit is derived for continuously parameterized families of channels admitting only fixed points. It is shown that the spectral gap of $\mathcal{A}_{\boxplus}$ can be closed for any segment of the adiabatic path without affecting the adiabatic evolution of the fixed points. The only gap that must remain open is that of $\mathcal{A}_{\mathbf{⿴}}$.

\subsection{From channels to Lindbladians}

Consider a continuously parameterized family of channels $\mathcal{A}^{(s)}$ with $s \in[0,1]$ and with no rotating points. Instead of thinking of these as repeated applications of random channels [83, 84], we assume that $\mathcal{A}^{(s)}$ is smoothly varying. Starting with an initially fixed-point state of $\rho_{\infty}=\mathcal{A}^{(0)}\left(\rho_{\infty}\right)$, we determine how this state evolves under the map

$$
\mathcal{T}\left(\rho_{\infty}\right) \equiv \lim _{N \rightarrow \infty} \mathcal{A}^{(1)} \cdots \mathcal{A}^{\left(\frac{1}{N}\right)} \mathcal{A}^{\left(\frac{0}{N}\right)}\left(\rho_{\infty}\right)
$$

This is a discrete channel version of the usual quantum-mechanical adiabatic limit [85, 86], where the generator of unitary time evolution is slowly varied during evolution. Those familiar with unitary quantum mechanics would expect that, upon evolution in a closed path $\mathcal{A}^{(1)}=\mathcal{A}^{(0)}$, one remains in the fixed-point subspace of $\mathcal{A}^{(s)}$ and returns to the same state up to a geometric phase [87] or unitary matrix [88] operation. Such a limit has indeed been extended to fixed-point subspaces of Lindbladians (e.g., [89, Thm. 2.6]), and the few simple manipulations below will convert the above limit of discrete maps into this case. However, note that initializing in a subspace other than the one of slowest decay is not as straightforward [90-93].

To reduce the above, we use the calculus of differences [94]. For $n \in\{0,1, \cdots, N\}$, define the map $\rho^{\left(\frac{n+1}{N}\right)}=\mathcal{A}^{\left(\frac{n+1}{N}\right)}\left(\rho^{\left(\frac{n}{N}\right)}\right)$. Taking the discrete derivative yields

$d \rho^{(n)} \equiv \frac{\rho^{\left(\frac{n+1}{N}\right)}-\rho^{\left(\frac{n}{N}\right)}}{1 / N}=N\left(\mathcal{A}^{\left(\frac{n+1}{N}\right)}-\mathcal{I}\right)\left(\rho^{\left(\frac{n}{N}\right)}\right)$

At this point, one can proceed to derive a discrete version of the adiabatic expansion of Ref. [89]. However, it is simpler to take the continuum limit directly, yielding the evolution equation $\frac{1}{N} \frac{d \rho^{(s)}}{d s}=\mathcal{L}^{(s)}\left(\rho^{(s)}\right)$, generated by the Lindbladian

$$
\mathcal{L}^{(s)}=\mathcal{A}^{(s)}-\mathcal{I} .
$$

Therefore, adiabaticity of Kraus map families $\mathcal{A}^{(s)}$ is solely determined by their corresponding Lindbladians $\mathcal{L}^{(s)}$. Using the aforementioned results [89], the asymptotic solution to eq. (35) is

$$
\mathcal{T}\left(\rho_{\infty}\right)=\prod_{s \in[0,1]} \mathcal{P}_{\mathcal{A}}^{(s)}\left(\rho_{\infty}\right)+O(1 / N),
$$

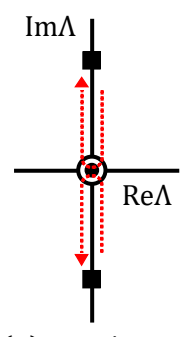

(a) Hamiltonian

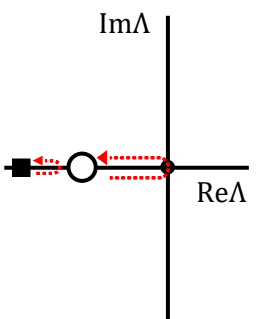

(b) Lindbladian
Figure 1: (a) The path $s \in[0,1]$ traced by the four eigenvalues $\Lambda$ of the superoperator corresponding to the Hamiltonian $H=\frac{1}{2} \cos ^{2} \pi s(|0\rangle\langle 0|-| 1\rangle\langle 1|)$ with eigenstates $|0\rangle,|1\rangle$. The two populations $|0\rangle\langle 0|$ (labeled by $\bullet$ ) and $|1\rangle\langle 1|(\bigcirc)$ are fixed points of the evolution while the two coherences $|0\rangle\langle 1|| 1\rangle,\langle 0|(\boldsymbol{\square})$ are eigenmatrices with the energy difference as the eigenvalue. As $|0\rangle$ and $|1\rangle$ become degenerate at $s=\frac{1}{2}$, the coherence eigenvalues go to zero, and so transitions between $|0\rangle$ and $|1\rangle$ can occur. (b) A path traced by the four eigenvalues of the Lindbladian with jump operator (41). The coherences remain separated from the origin when both $|0\rangle\langle 0|$ and $|1\rangle\langle 1|$ are fixed points at $s=\frac{1}{2}$, so transitions between $|0\rangle$ and $|1\rangle$ are suppressed.

where $\prod$ is the time-ordered product of $\mathcal{P}_{\mathcal{A}}^{(s)}$. The evolution can also be calculated using the Berry connection [59, 95]

$$
A_{\alpha, \mu \nu}=\operatorname{Tr}\left\{J^{\mu \dagger} \partial_{\alpha} \Psi_{\nu}\right\},
$$

where one can parameterize $\partial_{s}=\sum_{\alpha} v_{\alpha} \partial_{\alpha}$ into various directions $\alpha$ in parameter space with velocities $v_{\alpha}$, and $\{\Psi, J\}$ are the fixed points and conserved quantities, respectively. This connection allows us to express the closed-loop adiabatic evolution as a holonomy [96]

$$
\mathcal{T}\left(\rho_{\infty}\right)=\mathbb{P} e^{-\sum_{\alpha} \int d \alpha A_{\alpha}}\left(\rho_{\infty}\right)+O(1 / N),
$$

telling us how a state in the instantaneous fixed-point subspace has rotated as the entire subspace is parallel transported in the parameter space (with $\mathbb{P}$ denoting a path-ordered integral).

Now let us add in the $\boxplus$ structure of $\mathcal{A}$, meaning that for each $s$ there is an additional instantaneous set of projections $\mathcal{P}_{\boxplus}^{(s)}$. Assuming that all fixed points lie in $\boldsymbol{\Psi}$, it was shown [59] that the $O(1 / N)$ correction term depends on the spectrum of $\mathcal{L}_{\mathbb{\Xi}}$ and not of $\mathcal{L}_{\boxplus}$. Below, we go further and show that the dissipative gap of $\mathcal{L}_{\mathbb{⿴}}$ - the nonzero eigenvalue with the smallest real part - can even be closed without affecting the adiabatic evolution of the fixed points in

\subsection{The gap can close: a simple example}

Let us first consider a simple example of a channel whose gap in $\boxplus$ closes without affecting adiabatic evolution in $\boldsymbol{\oplus}$. Let $\mathcal{L}^{(s)}$ be a Lindbladian family with a single jump operator

$$
F^{(s)}=\left(\begin{array}{cc}
0 & \cos ^{2} \pi s \\
0 & 2
\end{array}\right),
$$


where we have written the matrix in the $|0\rangle,|1\rangle$ basis $(\boldsymbol{\Xi}=|0\rangle\langle 0|, \boldsymbol{\Xi}=| 0\rangle\langle 1|$, etc. $)$. This system has a fixed point $\Psi=|0\rangle\langle 0|$, two coherences $|0\rangle\langle 1|| 1\rangle,\langle 0|$ with eigenvalue $-\frac{1}{2}\left(4+\cos ^{4} \pi s\right)$, and one eigenmatrix $\psi$ with presence in $\boxplus$ and eigenvalue $-\cos ^{4} \pi s$. As $s \rightarrow \frac{1}{2}$, the gap of $\mathcal{L}_{\mathbb{⿴}}^{(s)}$ closes and $\psi \rightarrow|1\rangle\langle 1|$ becomes a fixed point. However, the two coherences do not become fixed, as their eigenvalues are -2 at $s=\frac{1}{2}$. This could not have been possible in the case of a Hamiltonian (see Fig. 1), where coherences between two degenerate eigenstates are always fixed points. Since no coherences are there to facilitate transitions between $|0\rangle$ and $|1\rangle$, a state in $|0\rangle\langle 0|$ remains there even at $s=\frac{1}{2}$.

\subsection{General case}

The degeneracy in the example below occurs only at a point, and it has already been shown that point crossings do not affect the adiabatic limit [86, 97]. Here, I show that the adiabatic evolution of $\boldsymbol{\Psi}$ is not affected by $\boxplus$ even if the gap of $\mathcal{L}_{\boxplus}^{(s)}$ is closed for a finite segment of $s$. Namely, adiabatic evolution (40) of a state initially in $\Theta$ is

$$
\mathcal{T}\left(\rho_{\infty}\right)=\prod_{s \in[0,1]} \mathcal{P}_{\mathcal{E}}^{(s)}\left(\rho_{\infty}\right)+O(1 / N),
$$

where $\mathcal{P}_{\mathcal{E}}^{(s)}$ is the asymptotic projection of the faithful Lindbladian

$$
\mathcal{L}_{\boxplus}^{(s)}=\left(\mathcal{A}^{(s)}-\mathcal{I}\right)_{\boxplus}=\mathcal{E}^{(s)}-\mathcal{P}_{\boxplus}^{(s)} .
$$

This more general statement holds if, for all $s, \mathcal{L}_{\mathbf{W}}^{(s)}$ is gapped and the dimension of the fixed-point subspace located in $\boxplus$ is constant $\left(\partial_{s} \operatorname{TR}\left\{\mathcal{P}_{\mathcal{E}}^{(s)}\right\}=0\right)$. Thus, the previous result [59, Eq. (5.16)] can be extended to cases where $\mathcal{L}_{\boxplus}^{(s)}$ also has fixed points. To show this, we need to consider two cases: case I (II) analyzing any segment of $s$ during which the gap of $\mathcal{L}_{\boxplus}^{(s)}$ is closed (open).

For case I, we apply the usual adiabatic theorem for the fixed-point subspace of $\mathcal{L}^{(s)}$. Therefore, adiabatic evolution is governed by the Berry connection $A$ (39). Suppressing $s$, let $\{\psi, j\}$ be any fixed point and its corresponding conserved quantity of $\mathcal{L}_{\boxplus}$. Let $\{\Psi, J\}$ be any such pair for $\mathcal{L}_{\boldsymbol{\Xi}}$. Since we start in a state $\rho_{\infty} \in \boxplus$, we need to consider whether there are any transitions into $\boxplus$ caused by the terms $\operatorname{Tr}\left\{j^{\dagger} \partial_{\alpha} \Psi\right\}$ of $A$. We know from Prop. 3 that $j$ may only overlap with the fixed-point space of $\mathcal{L}_{\boxplus}$ and with some part of any decaying subspace. Since $\square$ is not a decaying subspace, $j=\mathcal{P}_{\Xi}(j)$. Moreover, since the derivative $\partial_{\alpha}$ cannot map $\boxminus$ into $\boxplus,{ }^{2}$ we have that $\partial_{\alpha} \Psi=\mathcal{P}_{\mathbf{⿴}}\left(\partial_{\alpha} \Psi\right)$.

${ }^{2}$ This is because of a so-called no-leak condition [59]: for any $\left|\phi_{1}\right\rangle\left\langle\phi_{2}\right| \in \boxminus, \partial_{\alpha}$ acts on either the ket or the bra part by the product rule and never on both at the same time. Therefore, $\partial_{\alpha}\left(\left|\phi_{1}\right\rangle\left\langle\phi_{2}\right|\right) \in$
Combining these yields

$$
\operatorname{Tr}\left\{j^{\dagger} \partial_{\alpha} \Psi\right\}=\operatorname{Tr}\left\{\mathcal{P}_{\mathbb{\boxplus}}\left(j^{\dagger}\right) \mathcal{P}_{\mathbf{\Psi}}\left(\partial_{\alpha} \Psi\right)\right\}=0,
$$

meaning that $A$ does not contain any transitions of $\boldsymbol{\Psi}$ fixed points into those in $\boxminus$. This is true more generally, namely, for the decomposition of the fixed-point set into blocks indexed by $\varkappa$ discussed in Sec. 5.4 , each block evolves separately in the adiabatic limit and no transitions between different $\varkappa$ are allowed.

For case II, $\mathcal{L}_{\boxplus}$ is always gapped and so adiabatic evolution is determined by the Berry connection of the fixed points of $\mathcal{L}_{\boldsymbol{\Xi}}$. Therefore, in the adiabatic limit, a state in $\boxplus$ will remain in the instantaneous $\boxplus$ as $s$ is slowly varied. Moreover, one can instead consider only $\mathcal{P}_{\mathcal{E}}$ because the derivative operator cannot map $\boxplus$ into $\boxplus,^{2}$

$$
\operatorname{Tr}\left\{J^{\dagger} \partial_{\alpha} \Psi\right\}=\operatorname{Tr}\left\{\mathcal{P}_{\mathbf{⿴}}\left(J^{\dagger}\right) \mathcal{P}_{\mathbf{\Psi}}\left(\partial_{\alpha} \Psi\right)\right\}=\operatorname{Tr}\left\{J_{\mathbb{\Theta}}^{\dagger} \partial_{\alpha} \Psi\right\} .
$$

Thus, in both of the above cases, one only had to care about the parts of the Berry connection $A$ consisting of the fixed points and conserved quantities of $\mathcal{L}_{\boldsymbol{\Psi}}$ (43). Therefore, the adiabatic evolution can be expressed using only the associated projection $\mathcal{P}_{\mathcal{E}}$ (42).

\section{Application: Matrix product states}

For those who skimmed Secs. 3-6, those parts focused on the distinction between a channel $\mathcal{A}$ and its corresponding faithful channel $\mathcal{E} \equiv \mathcal{P}_{\boldsymbol{G}} \mathcal{A} \mathcal{P}_{\boldsymbol{⿴}}-\mathcal{A}$ restricted to the largest invariant subspace $\boxplus$. The block $\boxplus$ thus forms a decaying subspace, but the asymptotic projection $\mathcal{P}_{\mathcal{A}}(10)$ of $\mathcal{A}=\left\{A^{\ell}\right\}$ nevertheless retains information from states in $\boxminus$ by transferring it into $\boxplus$ through the operators $A_{\boxplus}^{\ell}$. A similar effect is observed in the thermodynamic limit of matrix product states (MPS). Here, the results about $\mathcal{A}$ are applied to obtain an unambiguous thermodynamic limit for any MPS that is translationally invariant in the bulk, but has non-trivial boundary effects. Then, I show how one can absorb any dependence of said limit on the decaying parts $\boxminus$ of the bond degrees of freedom into the boundary conditions $B$. This allows one to shorten the bond dimension and use the $\mathcal{A}_{\square}=\mathcal{E}$ instead of the full $\mathcal{A}$.

Proposition 4. Let $\left|\Phi_{\mathcal{A}}^{\{B\}}\right\rangle$ be an MPS with transfer channel $\mathcal{A}$ and boundary matrix $B$. Let $O$ be an operator on a single site of a chain of length $M$. Then, there exists a $\widetilde{B}$ such that

$$
\lim _{M \rightarrow \infty}\left\langle\Phi_{\mathcal{A}}^{\{B\}}|O| \Phi_{\mathcal{A}}^{\{B\}}\right\rangle=\lim _{M \rightarrow \infty}\left\langle\Phi_{\mathcal{E}}^{\{\widetilde{B}\}}|O| \Phi_{\mathcal{E}}^{\{\widetilde{B}\}}\right\rangle,
$$

where $\mathcal{E}=\mathcal{A}_{\boxplus}$ is the restriction of $\mathcal{A}$ to the maximal invariant subspace $\boldsymbol{\boxplus}$.

A similar result holds for two single-site observables that are infinitely far apart in the chain and infinitely 
far from the boundaries. The proof consists of the remainder of this section. Due to the aforemenetioned effects, $\widetilde{B} \neq B_{\mathbf{q}}$; an expression for $\widetilde{B}$ in terms of $B$ is derived below. The technique is somewhat reverse of what has been done before (see Sec. 3.2.2 of [3]): instead of first considering a general MPS, I simplify the corresponding MPS of a general faithful channel $\mathcal{E}$ in the thermodynamic limit, and then show how any contribution from its extension $\mathcal{A}$ can be absorbed into the boundary.

\subsection{MPS from faithful channels}

Our playground is now a one-dimensional chain of $2 M+1$ spins. Each spin is $L$-dimensional and indexed by the physical index $\ell$. Let us consider a faithful channel $\mathcal{E}=\left\{E_{\ell}\right\}_{\ell=1}^{L}$ (with $E_{\ell} N \times N$ matrices for some bond dimension $N$ ) and write its corresponding $\operatorname{MPS}\left|\Phi_{\mathcal{E}}^{\{B\}}\right\rangle:^{3}$

$$
\left|\Phi_{\mathcal{E}}^{\{B\}}\right\rangle \propto \sum_{\ell_{-M}, \cdots, \ell_{M}=0}^{L-1} \operatorname{Tr}\left\{B E_{\ell_{-M}} \cdots E_{\ell_{M}}\right\}\left|\ell_{-M} \cdots \ell_{M}\right\rangle,
$$

where the $N \times N$ boundary matrix $B$ provides the ability to pin the boundary of the chain to various states [98]. Physically meaningful boundaries are either $B=I$ (the identity) for translationally invariant MPS's or $B=|r\rangle\langle l|$ for some states $|r\rangle,|l\rangle$ quantifying the effect of the boundary on the right and left ends of the chain. I consider cases where the bond dimension $N$ is independent of system size $M$, noting there are interesting cases where this is not so [3, Appx. A.1].

The Kraus operators of $\mathcal{E}$ decompose into blocks $\varkappa$, as in eq. (34), with each block a noiseless subsystem. Since the Kraus operators are block-diagonal, $\operatorname{Tr}\left\{B E_{\ell_{-M}} \cdots E_{\ell_{M}}\right\}$ decouples into a sum of traces over each block. Each block corresponds to its own MPS, and the different MPS will not overlap with each other in the thermodynamic limit $(M \rightarrow \infty)$. Thus, we can consider only one block from now on. In this block, the Kraus operators factor as $E_{\ell}=U \otimes R_{\ell}$, where $\left\{R_{\ell}\right\}$ is an irreducible channel. The DFS part $U$ can be diagonalized, so the MPS once again decouples. This leaves us with only the irreducible parts $R_{\ell}$ and automatically puts our MPS into canonical form $[3,37]$. We assume from now on that $\mathcal{E}$ is irreducible.

One can obtain the normalization of the MPS in the thermodynamic limit by tracing out the sites one by one:

$$
\begin{aligned}
\lim _{M \rightarrow \infty}\left\langle\Phi_{\mathcal{A}}^{\{B\}} \mid \Phi_{\mathcal{A}}^{\{B\}}\right\rangle & =\lim _{M \rightarrow \infty} \operatorname{TR}\left\{\mathcal{E}^{\alpha(2 M+1)} \mathcal{B}\right\} \\
& =\operatorname{TR}\left\{\mathcal{P}_{\mathcal{E}} \mathcal{B}\right\}
\end{aligned}
$$

${ }^{3}$ Since applying identical transformations $U$ to each site is the same as changing basis for the Kraus operators of $\mathcal{E}, E_{\ell} \rightarrow$ $\sum_{\ell^{\prime}} U_{\ell \ell^{\prime}} E_{\ell^{\prime}}$, more technically this is a study of sets of MPS related by local unitaries. where $\mathcal{B} \equiv B \otimes \bar{B}$, the trace is over superoperator space, and $\alpha$ is the parameter that eliminates phases stemming from rotating points. We assume $\mathcal{E}$ has $N$ rotating points, meaning that all peripheral eigenvalues are $N$ th roots of unity. Thus, setting $\alpha=N$ yields an unambiguous thermodynamic limit for general boundary conditions. ${ }^{4}$ Similarly, the expectation value of an observable $O$ on a site in this thermodynamic limit is

$$
\lim _{M \rightarrow \infty}\left\langle\Phi_{\mathcal{E}}^{\{B\}}|O| \Phi_{\mathcal{E}}^{\{B\}}\right\rangle=\operatorname{Tr}\left\{\mathcal{P}_{\mathcal{E}} \mathcal{O} \mathcal{P}_{\mathcal{E}} \mathcal{B}\right\}
$$

where the corresponding superoperator is

$$
\mathcal{O} \equiv \sum_{k, \ell=0}^{L-1}\langle\ell|O| k\rangle E_{k} \otimes \overline{E_{\ell}}
$$

Using what we know about $\mathcal{P}_{\mathcal{E}}(31)$ for irreducible channels and plugging in eqs. (51) and (71), eq. (50) becomes

$$
\begin{aligned}
\lim _{M \rightarrow \infty} & \left\langle\Phi_{\mathcal{E}}^{\{B\}}|O| \Phi_{\mathcal{E}}^{\{B\}}\right\rangle=N^{2} \sum_{k, \ell=0}^{L-1}\langle\ell|O| k\rangle \times \\
& \times \sum_{\mu \in \mathbb{Z}_{N}} \operatorname{Tr}\left\{\Pi_{\mu} E_{k} \Pi_{\mu+1} \varrho E_{\ell}^{\dagger}\right\} \operatorname{Tr}\left\{\Pi_{\mu+1} B \Pi_{\mu} \varrho B^{\dagger}\right\}
\end{aligned}
$$

Each term in the sum over $\mu$ is determined by the boundary block $\Pi_{\mu+1} B \Pi_{\mu}$, and the unique structure of $\mathcal{E}$ implies that all other parts of $B$ are irrelevant in the thermodynamic limit. In fact, each term in the sum corresponds to a contribution coming from a distinct MPS. Another way to see this is by taking the lattice size $M$ to be a multiple of $N$ and performing the periodic decomposition [3, Thm. 5].

\subsection{Adding decay}

Now let us extend $\mathcal{E}$ to $\mathcal{A}$, meaning that the MPS $\left|\Phi_{\mathcal{A}}^{\{B\}}\right\rangle$ is the same eq. (47) but with $E_{\ell} \rightarrow A^{\ell}$. After some algebra, the coefficient $\operatorname{Tr}\left\{B\left(A^{\ell_{-M}} \cdots A^{\ell_{M}}\right)\right\}$ becomes equal to

$$
\begin{gathered}
\operatorname{Tr}\left\{B_{\boxplus}\left(E_{\ell_{-M}} \cdots E_{\ell_{M}}\right)\right\}+\operatorname{Tr}\left\{B_{\boxplus}\left(A_{\boxplus}^{\ell_{-M}} \cdots A_{\boxplus}^{\ell_{M}}\right)\right\} \\
+\sum_{m=-M}^{M} \operatorname{Tr}\left\{B_{\boxplus}\left(E_{\ell_{-M}} \cdots E_{\ell_{m-1}}\right) A_{\boxplus}^{\ell_{m}}\left(A_{\boxplus}^{\ell_{m}} \cdots A_{\boxplus}^{\ell_{M}}\right)\right\} .
\end{gathered}
$$

This is precisely the same structure occurring in, e.g., product vacua with boundary states $[31,32]$. The first term corresponds to the MPS $\left|\Phi_{\mathcal{E}}^{\{B\}}\right\rangle$ we already studied. The second term vanishes in the thermodynamic limit because its corresponding transfer matrix does

${ }^{4}$ Note that $\left|\Psi_{\mathcal{P}_{\mathcal{E}}}^{\{B\}}\right\rangle$ is also the fixed-point MPS that $\left|\Phi_{\mathcal{E}}^{\{B\}}\right\rangle$ flows to under RG transformations [99-101], and $\lim _{M \rightarrow \infty}\left\langle\Phi_{\mathcal{E}}^{\{B\}} \mid \Phi_{\mathcal{E}}^{\{B\}}\right\rangle=\left\langle\Psi_{\mathcal{P}_{\mathcal{E}}}^{\{B\}} \mid \Psi_{\mathcal{P}_{\mathcal{E}}}^{\{B\}}\right\rangle$, so simplifying $\mathcal{P}_{\mathcal{E}}$ also yields insight into the structure of RG fixed points. 
not have any fixed points. When $B_{\mathbb{\Xi}} \neq 0$, the third term is present and has the form of a translationallyinvariant domain wall excitation. Therefore, the decaying subspace $\boxminus$ corresponds to extra degrees of freedom on each site which house such an excitation. This excitation is never present for periodic boundary conditions $(B=I)$, allowing one to straightforwardly derive an standard form for that case [3, 37]. However, here we focus on "twisted" boundaries $B_{\boldsymbol{\nabla}} \neq 0$.

The single-site expectation value (50) is now

$$
\lim _{M \rightarrow \infty}\left\langle\Phi_{\mathcal{A}}^{\{B\}}|O| \Phi_{\mathcal{A}}^{\{B\}}\right\rangle=\operatorname{TR}\left\{\mathcal{P}_{\mathcal{E}} \mathcal{O} \mathcal{P}_{\mathcal{A}} \mathcal{B}\right\}
$$

where the second projection is now $\mathcal{P}_{\mathcal{A}}$ due to the presence of the boundary term $\mathcal{B}_{\boldsymbol{m}}$. Simplifying, the analogue of eq. (52) is then

$$
\begin{aligned}
& \lim _{M \rightarrow \infty}\left\langle\Phi_{\mathcal{A}}^{\{B\}}|O| \Phi_{\mathcal{A}}^{\{B\}}\right\rangle=N^{2} \sum_{k, \ell=0}^{L-1}\langle\ell|O| k\rangle \times \\
& \times \sum_{\mu \in \mathbb{Z}_{N}} \operatorname{Tr}\left\{\Pi_{\mu} E_{k} \Pi_{\mu+1} \varrho E_{\ell}^{\dagger}\right\} \operatorname{Tr}\left\{\Pi_{\mu+1} \mathcal{P}_{\mathcal{A}}\left(B \Pi_{\mu} \varrho B^{\dagger}\right)\right\} .
\end{aligned}
$$

Now there are two contributions from $\mathcal{P}_{\mathcal{A}}, \mathcal{P}_{\mathcal{E}}$ and $\mathcal{P}_{\mathcal{A}} \mathcal{P}_{\mathbb{\Xi}}$, with the latter determined by Prop. 3. However, it is possible to absorb both into a new boundary matrix $\widetilde{B}$ such that the expectation value in $\left|\Phi_{\mathcal{E}}^{\{\widetilde{B}\}}\right\rangle$ is the same as that of $\left|\Phi_{\mathcal{A}}^{\{B\}}\right\rangle$. To determine $\widetilde{B}$, note that for eqs. (52) (with $B \rightarrow \widetilde{B}$ ) and (55) to be equal, we must have

$$
\operatorname{Tr}\left\{\Pi_{\mu+1} \widetilde{B} \Pi_{\mu} \varrho \widetilde{B}^{\dagger}\right\}=\operatorname{Tr}\left\{\Pi_{\mu+1} \mathcal{P}_{\mathcal{A}}\left(B \Pi_{\mu} \varrho B^{\dagger}\right)\right\} \equiv x_{\mu} .
$$

This $x_{\mu} \geq 0$ since $B \Pi_{\mu} \varrho B^{\dagger}$ is positive, $\mathcal{P}_{\mathcal{A}}$ is a channel, and $\Pi_{\mu+1}$ is a projection. Now, write out the projections, $\Pi_{\mu}=\sum_{\xi}|\mu, \xi\rangle\langle\mu, \xi|$ (with $\xi$ depending on $\mu$ ), to obtain

$$
\operatorname{Tr}\left\{\Pi_{\mu+1} \widetilde{B} \Pi_{\mu} \varrho \widetilde{B}^{\dagger}\right\}=\sum_{\xi} \varrho_{\mu, \xi} \sum_{\xi^{\prime}}\left|\left\langle\mu+1, \xi^{\prime}|\widetilde{B}| \mu, \xi\right\rangle\right|^{2},
$$

where $\varrho_{\mu, \xi} \equiv\langle\mu, \xi|\varrho| \mu, \xi\rangle>0$ (since $\varrho$ is full-rank). Therefore, setting

$$
\left\langle\mu+1, \xi|\widetilde{B}| \mu, \xi^{\prime}\right\rangle=\sqrt{N \frac{x_{\mu}}{\operatorname{Tr}\left\{\Pi_{\mu+1}\right\}}}
$$

satisfies the equality (56). (We used $\operatorname{Tr}\left\{\Pi_{n} \varrho\right\}=1 / N$; see Sec. 5.2.) Thus, we have shown how to construct a $\widetilde{B}$ that satisfies eq. (46).

\subsection{Other observables}

The same result occurs with two observables $O^{(1)}$ and $O^{(2)}$ (with corresponding superoperators $\mathcal{O}^{(1)}$ and $\left.\mathcal{O}^{(2)}\right)$ separated by some number of sites $W$,

$\lim _{M \rightarrow \infty}\left\langle\Phi_{\mathcal{A}}^{\{B\}}\left|O^{(1)} O^{(2)}\right| \Phi_{\mathcal{A}}^{\{B\}}\right\rangle=\operatorname{Tr}\left\{\mathcal{P}_{\mathcal{A}} \mathcal{O}^{(1)} \mathcal{A}^{W} \mathcal{O}^{(2)} \mathcal{P}_{\mathcal{A}} \mathcal{B}\right\}$ and take the $W \rightarrow \infty$ limit by blocking sites in order to get rid of any phases from rotating points. This yields

$\lim _{M, W \rightarrow \infty}\left\langle\Phi_{\mathcal{A}}^{\{B\}}\left|O^{(1)} O^{(2)}\right| \Phi_{\mathcal{A}}^{\{B\}}\right\rangle=\operatorname{Tr}\left\{\mathcal{P}_{\mathcal{E}} \mathcal{O}^{(1)} \mathcal{P}_{\mathcal{E}} \mathcal{O}^{(2)} \mathcal{P}_{\mathcal{A}} \mathcal{B}\right\}$.

After the simplifications of the previous section, we observe that in order for $\Phi_{\mathcal{E}}^{\{\widetilde{B}\}}$ to have the same expectation values, one now has to tune the "next nearestneighbor" elements $\operatorname{Tr}\left\{\Pi_{\mu+2} \widetilde{B} \Pi_{\mu} \varrho \widetilde{B}^{\dagger}\right\}$ [independent from $x_{\mu}$ (56) for $N \geq 3$ ]. The potentially interesting case of $N=2$ is left for future investigation.

Similarly, consider an observable touching the left boundary:

$$
\lim _{M \rightarrow \infty}\left\langle\Phi_{\mathcal{A}}^{\{B\}}\left|O^{(L)}\right| \Phi_{\mathcal{A}}^{\{B\}}\right\rangle=\operatorname{TR}\left\{\mathcal{O}^{(L)} \mathcal{P}_{\mathcal{A}} \mathcal{B}\right\}
$$

Somewhat surprisingly, considering an observable touching the right boundary produces something completely different:

$$
\lim _{M \rightarrow \infty}\left\langle\Phi_{\mathcal{A}}^{\{B\}}\left|O^{(R)}\right| \Phi_{\mathcal{A}}^{\{B\}}\right\rangle=\operatorname{TR}\left\{\mathcal{P}_{\mathcal{A}} \mathcal{O}^{(R)} \mathcal{B}\right\}
$$

Notice how $\mathcal{P}_{\mathcal{A}}$ now comes before the observable [cf. the first equality of Eq. (61)], which results in a series of new terms stemming from combinations of $A_{\boxplus}^{\ell}$ and $A_{\mathbb{⿴}}^{\ell}$ with $B$. Why is there an asymmetry between the two boundaries? This has to do with the fact that we had initially assumed an asymmetric form for our MPS, $A^{\ell}=A_{\mathbf{a}}^{\ell}$. The domain wall-type excitations represented by the third term in Eq. (53) are such that there is always a $A_{\boxplus}$ at the right-most site $M$.

\section{Conclusion}

An important property of quantum channels $\mathcal{A}$ is their asymptotics, i.e., their behavior in the limit of infinite applications, akin to the infinite-time limit of Lindbladians $[8,59]$. An infinite product of $\mathcal{A}$ produces the channel's asymptotic projection $\mathcal{P}_{\mathcal{A}}-$ a projection on all of the non-decaying eigenspaces of the channel (i.e., whose eigenvalues have unit modulus). The superoperator $\mathcal{P}_{\mathcal{A}}$ can be constructed out of the channel's left and right rotating points, or as they are called here, conserved quantities $J$ and steady-state basis elements $\Psi$. The aim of the first half of this work is to determine which parts of an initial state are conserved in the limit of infinite applications of the channel, extending analogously motivated work for Lindbladian channels [8]. This involves a derivation of the structure of both the $\Psi$ (already known) and the $J$ (developed here) making up $\mathcal{P}_{\mathcal{A}}$.

I start off with two statements about channels admitting a full-rank fixed point, which I call faithful. The first is that any $J$ commute with a faithful channel's Kraus operators up to a phase. The second is that the eigenvalue of any diagonalizable $J$ of a faithful channel is an $N$ th root of unity, where $N$ is tightly 
bounded by trace norm of $J$. A third result deals with determining the dependence of the asymptotic state on the initial state and on properties of $\mathcal{A}$. An analytical formula is derived that quantifies the dependence of the final state on initial states located in $\mathcal{A}$ 's decaying eigenspaces (i.e., whose eigenvalues are less than one in modulus).

The aim of the second half of this work is to apply results from the first half to an adiabatic limit for channels and to matrix product states. In both applications, it is shown that the channel $\mathcal{E}$ - the restriction of $\mathcal{A}$ to its largest invariant subspace - is sufficient to work with for the outlined purposes. An adiabatic limit for channels in developed, and it is shown that the gap of the part of the channel acting on the decaying subspace can close. The second application is to matrix product states (MPS), where asymptotics come into play in the thermodynamic limit or in the limit of infinite renormalization transformations. In the same way that asymptotic states depend on initial states, the thermodynamic limit of MPS (whose transfer matrices admit more than one fixed point) depends on the boundary conditions. In such situations, the effects of any decaying bond degrees of freedom can be absorbed in the boundary conditions. Quantitatively, it is shown that the thermodynamic expectation value of a local operator $O$ with an MPS having transfer matrix $\mathcal{A}$ and boundary condition $B$ is equivalent to the expectations values with MPS having transfer matrix $\mathcal{E}$ and a modified boundary condition $\widetilde{B}(46)$. This allows one to remove extra bond degrees of freedom when considering the thermodynamic limit. Since similar two-dimensional MPS (often called "PEPS" [102]) also correspond to a transfer channel, such techniques may be further generalized to study PEPS dependence on boundaries.

\section{Acknowledgments}

Insightful discussions with B. Bradlyn, X. Chen, M. Fraas, L. Jiang, D. Perez-Garcia, M. B. Sahinoglu, N. Schuch, F. Ticozzi, A. M. Turner, and M. M. Wolf are acknowledged. This research was supported in part by the National Science Foundation (PHY17-48958) and the Walter Burke Institute for Theoretical Physics at Caltech. I thank KITP Santa Barbara for their hospitality as part of the Quantum Physics of Information workshop.

\section{A Proofs}

Proposition 1. Let $\mathcal{E}=\left\{E_{\ell}\right\}$ be a faithful channel. Let $J$ be a conserved quantity of $\mathcal{E}$, i.e., $\mathcal{E}^{\ddagger}(J)=$ $e^{-i \Delta} J$ for some real $\Delta$. Then, for all $\ell$,

$$
J E_{\ell}=e^{-i \Delta} E_{\ell} J
$$

Proof. This result reduces to known results for irreducible [48, Thm. 4.2], ergodic [27, Thm. 9], or unital [103, Thm. 4] channels. Another proof is using Thms. 4.1-4.2 and Corollary 4.3 in Ref. [26]. This proof extends an often-used [24, 62] application of the dissipation function [19] from fixed points to rotating points. An analogous extension for Lindbladians is in Ref. [59]. Let

$$
X_{\ell} \equiv J E_{\ell}-e^{-i \Delta} E_{\ell} J
$$

for each Kraus operator $E_{\ell}$. Then, after some algebra,

$$
\sum_{\ell} X_{\ell}^{\dagger} X_{\ell}=\mathcal{E}^{\ddagger}\left(J^{\dagger} J\right)-J^{\dagger} J
$$

Now multiply both sides by a full-rank fixed point $\rho_{\text {ss }}$ and take the trace. Moving $\mathcal{E}^{\ddagger}$ under the HilbertSchmidt inner product so that it acts on $\rho_{\text {ss }}$ yields

$$
\operatorname{Tr}\left\{\mathcal{E}\left(\rho_{\mathrm{ss}}\right) J^{\dagger} J\right\}-\operatorname{Tr}\left\{\rho_{\mathrm{ss}} J^{\dagger} J\right\}=0
$$

for the right-hand side, meaning that

$$
\sum_{\ell} \operatorname{Tr}\left\{\rho_{\mathrm{ss}} X_{\ell}^{\dagger} X_{\ell}\right\}=0
$$

Since $X_{\ell}^{\dagger} X_{\ell} \geq 0$ and $\rho_{\mathrm{ss}}>0$, the only way for the above to hold is for $X_{\ell}=0$, which implies the statement.

Proposition 2. Let $\mathcal{E}=\left\{E_{\ell}\right\}$ be a faithful channel. Let $J_{\mathrm{dgn}}$ be such that $\mathcal{E}^{\ddagger}\left(J_{\mathrm{dgn}}\right)=e^{-i \Delta} J_{\mathrm{dgn}}$ for some real $\Delta$ and assume $J_{\mathrm{dgn}}$ is diagonalizable. Then, there exists an integer $n$ such that

$$
\Delta=\frac{2 \pi}{N} n \quad \text { for some } \quad N \leq\left\|J_{\mathrm{dgn}}\right\|_{1},
$$

where \|\|$_{1}$ is the trace norm.

Proof. The tighter bound compelements similar work ([29]; [23], Thm. 6.6; [2], Prop. 3.3; [103], Corr. 3; [46], Prop. 28). First, there must exist an $N \geq 1$ such that

$$
\Pi \equiv J_{\mathrm{dgn}}^{N}
$$

is a projection $\left(J_{\mathrm{dgn}}^{2 N}=J_{\mathrm{dgn}}^{N}\right)$. To show this, assume by contradiction that all powers of $J_{\mathrm{dgn}}$ are distinct. Then, there is an infinite sequence of conserved quantities $J_{\mathrm{dgn}}^{N}$ with eigenvalues $e^{-i N \Delta}$ due to Prop. 1. But the Hilbert space is $D$-dimensional, so there are at most $D^{2}$ fixed/rotating points. Moreover, $e^{-i N \Delta}=1$; otherwise, $J_{\mathrm{dgn}}^{N}$ would have a different eigenvalue than $J_{\mathrm{dgn}}^{2 N}$. Therefore, there exists an $N \leq D^{2}$ such that $\Delta=\frac{2 \pi}{N} n$ for $n \in\{0,1, \cdots N-1\}$, i.e., $J_{\mathrm{dgn}}$ has eigenvalues which are $N$ th roots of unity.

Now I show that $N \leq\left\|J_{\text {dgn }}\right\|_{1}$. Since $J_{\text {dgn }}$ is diagonalizable, one can write

$$
J_{\mathrm{dgn}}=\sum_{n \in \mathbb{Z}_{N}} \omega^{n} \Pi_{n},
$$


where $\omega \equiv e^{i \frac{2 \pi}{N}}$ and the projections onto the eigenspace of $J_{\mathrm{dgn}}$ corresponding to eigenvalue $\omega^{n}$ are

$$
\Pi_{n}=\frac{1}{N} \sum_{k \in \mathbb{Z}_{N}} \omega^{-n k} J_{\mathrm{dgn}}^{k} .
$$

Proposition 1 implies that for all $\ell$,

$$
\Pi_{n} E_{\ell}=E_{\ell} \Pi_{n+1} \quad \text { modulo } N \text {. }
$$

Projecting $E_{\ell}$ onto the support of $J_{\mathrm{dgn}}$ using $\Pi=$ $\sum_{n} \Pi_{n}(68)$ and applying the above yields a matrix of exactly $N$ blocks $\Pi_{n} E_{\ell} \Pi_{n+1}$,

$$
\Pi E_{\ell} \Pi=\sum_{n \in \mathbb{Z}_{N}} \Pi_{n} E_{\ell} \Pi_{n+1} .
$$

In the basis of eigenstates of $\Pi$, this can be viewed as a matrix of dimension

$$
\operatorname{Tr} \Pi=\operatorname{Tr} J_{\mathrm{dgn}}^{N}=\left\|J_{\mathrm{dgn}}\right\|_{1} .
$$

Since $\Pi_{n}$ are distinct orthogonal projections, the largest number of blocks in $\Pi E_{\ell} \Pi$ occurs when all $\Pi_{n}$ are rank one; then $N=\left\|J_{\mathrm{dgn}}\right\|_{1}$. Generally, $N \leq\left\|J_{\mathrm{dgn}}\right\|_{1}$.

Proposition 3. The conserved quantities of $\mathcal{A}$ corresponding to eigenvalues $e^{i \Delta}$ are The conserved quantities of $\mathcal{A}$ corresponding to eigenvalues $e^{i \Delta}$ are

$$
J=J_{\boldsymbol{\Theta}}+J_{\boxplus}=J_{\boldsymbol{\Theta}}+\mathcal{R}_{\boldsymbol{\Phi}}^{(\Delta) \ddagger} \mathcal{A}^{\ddagger}\left(J_{\boldsymbol{\varpi}}\right),
$$

where $J_{\boldsymbol{\Xi}}$ are the conserved quantities of $\mathcal{A}_{\boldsymbol{\Xi}}=\mathcal{E}$ and

$$
\mathcal{R}_{\mathbb{\Xi}}^{(\Delta)}=-\left(\mathcal{A}-e^{i \Delta}\right)_{\mathbb{\Xi}}^{-1} .
$$

Proof. I generalize previous results ([24], Lemma 5.8; [71], Prop. 7) to the case of rotating points. Start by writing the eigenvalue equation

$$
J e^{-i \Delta}=\mathcal{A}^{\ddagger}(J)
$$

in terms of the four-corners decomposition of $J$ and

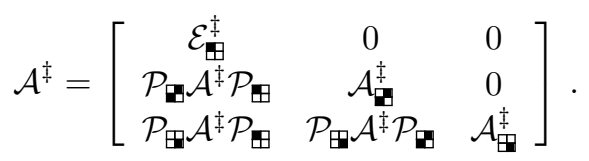

The three zeroes in the above decomposition for $\mathcal{A}^{\ddagger}$ can be derived by brute-force use of eq. (16). The eigenvalue equation is equivalent to

$$
\begin{aligned}
& J_{\boldsymbol{G}} e^{-i \Delta}=\mathcal{A}_{\boldsymbol{\Theta}}^{\dagger}\left(J_{\boldsymbol{\Theta}}\right) \\
& J_{\mathbf{\Xi}} e^{-i \Delta}=\mathcal{P}_{\boldsymbol{\Psi}} \mathcal{A}^{\ddagger}\left(J_{\boldsymbol{\Theta}}\right)+\mathcal{A}_{\mathbf{\Xi}}^{\ddagger}\left(J_{\boldsymbol{\Psi}}\right) \\
& J_{\boxplus} e^{-i \Delta}=\mathcal{P}_{\boxplus} \mathcal{A}^{\ddagger}\left(J_{\boldsymbol{\Theta}}\right)+\mathcal{P}_{\mathbb{\boxplus}} \mathcal{A}^{\ddagger}\left(J_{\mathbb{\Xi}}\right)+\mathcal{A}_{\mathbb{\boxplus}}^{\ddagger}\left(J_{\boxplus}\right) .
\end{aligned}
$$

First, let's look at the term (78b):

$$
\mathcal{P}_{\boldsymbol{\Psi}} \mathcal{A}^{\ddagger}\left(J_{\boldsymbol{\Theta}}\right)=\mathcal{P}_{\boldsymbol{\Theta}} \mathcal{A}^{\ddagger}\left(J_{\boldsymbol{\Psi}}\right)+\mathcal{P}_{\boldsymbol{\Xi}} \mathcal{A}^{\ddagger}\left(J_{\boldsymbol{\Theta}}\right) .
$$

Using eqs. (13) and (17), one can see that

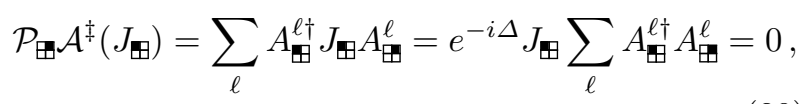

and similarly for the second term $\mathcal{P}_{\boldsymbol{\Phi}} \mathcal{A}^{\ddagger}\left(J_{\boldsymbol{E}}\right)$. This reduces eq. $(78 \mathrm{~b})$ to

$$
J_{\mathbf{m}} e^{-i \Delta}=\mathcal{A}_{\mathbf{q}}^{\ddagger}\left(J_{\mathbf{m}}\right) .
$$

This can in turn be used to show that $J_{\boldsymbol{m}}=0$ [59]. Assume by contradiction that $J_{\mathbb{*}} \neq 0$. Then, there must exist a corresponding right fixed point $\Psi$ with $\mathcal{A}_{\mathbf{\Psi}}(\Psi)=0$. But we have already assumed that all right fixed points are in $\boldsymbol{\Psi}$. Therefore, $J_{\boldsymbol{\Psi}}=0$.

The remaining eq. (78c) becomes

$$
J_{\mathbb{\Xi}} e^{-i \Delta}=\mathcal{P}_{\mathbb{\Xi}} \mathcal{A}^{\ddagger}\left(J_{\boldsymbol{\Xi}}\right)+\mathcal{A}_{\boxplus}^{\ddagger}\left(J_{\boxplus}\right)
$$

and can be used to solve for $J_{\mathbb{\nabla}}$ in terms of $J_{\mathbb{\Psi}}$ (since

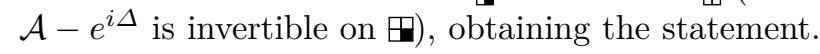

\section{References}

[1] Filippo Caruso, Vittorio Giovannetti, Cosmo Lupo, and Stefano Mancini. Quantum channels and memory effects. Rev. Mod. Phys., 86 (4):1203-1259, dec 2014. ISSN 0034-6861. DOI: 10.1103/RevModPhys.86.1203.

[2] M. Fannes, B. Nachtergaele, and R. F. Werner. Finitely correlated states on quantum spin chains. Commun. Math. Phys., 144(3):443490, mar 1992. ISSN 0010-3616. DOI: 10.1007/BF02099178.

[3] D. Perez-Garcia, F. Verstraete, M. M. Wolf, and J. I. Cirac. Matrix Product State Representations. aug. URL http://arxiv.org/abs/ quant-ph/0608197.

[4] V. Giovannetti, S. Montangero, and Rosario Fazio. Quantum Multiscale Entanglement Renormalization Ansatz Channels. Phys. Rev. Lett., 101(18):180503, oct 2008. ISSN 00319007. DOI: 10.1103/PhysRevLett.101.180503.

[5] Robert N. C. Pfeifer, Glen Evenbly, and Guifré Vidal. Entanglement renormalization, scale invariance, and quantum criticality. Phys. Rev. A, 79(4):040301, apr 2009. ISSN 1050-2947. DOI: 10.1103/PhysRevA.79.040301.

[6] Scott Aaronson, Mohammad Bavarian, and Giulio Gueltrini. Computability Theory of Closed Timelike Curves. sep 2016. URL http: //arxiv.org/abs/1609.05507.

[7] Ji-Woong Lee and Shashi Phoha. Operator sum representation for Markov transition models of human inference processes. In 2016 Am. Control Conf., pages 1590-1595. IEEE, jul 2016. ISBN 978-1-4673-8682-1. DOI: 10.1109/ACC.2016.7525143. 
[8] Victor V. Albert and Liang Jiang. Symmetries and conserved quantities in Lindblad master equations. Phys. Rev. A, 89(2):022118, feb 2014. ISSN 1050-2947. DOI: 10.1103/PhysRevA.89.022118.

[9] John Dengis, Robert Konig, and Fernando Pastawski. An optimal dissipative encoder for the toric code. New J. Phys., 16(1):013023, jan 2014. ISSN 1367-2630. DOI: 10.1088/13672630/16/1/013023.

[10] Seth Lloyd and Lorenza Viola. Engineering quantum dynamics. Phys. Rev. A, 65(1):010101, dec 2001. ISSN 1050-2947. DOI: 10.1103/PhysRevA.65.010101.

[11] B. Kraus, Hans Peter Büchler, S. Diehl, A. Kantian, A. Micheli, and P. Zoller. Preparation of entangled states by quantum Markov processes. Phys. Rev. A, 78(4):042307, oct 2008. ISSN 1050-2947. DOI: 10.1103/PhysRevA.78.042307.

[12] J. F. Poyatos, J Ignacio Cirac, and P. Zoller. Quantum Reservoir Engineering with Laser Cooled Trapped Ions. Phys. Rev. Lett., 77(23): 4728-4731, dec 1996. ISSN 0031-9007. DOI: 10.1103/PhysRevLett.77.4728.

[13] P. Schindler, Markus Müller, D. Nigg, J. T. Barreiro, E. A. Martinez, M. Hennrich, T. Monz, S. Diehl, P. Zoller, and R. Blatt. Quantum simulation of dynamical maps with trapped ions. Nat. Phys., 9(6):361-367, may 2013. ISSN 17452473. DOI: $10.1038 /$ nphys 2630.

[14] Shi-Jie Wei, Tao Xin, and Gui-Lu Long. Efficient universal quantum channel simulation in IBM's cloud quantum computer. Sci. China Physics, Mech. Astron., 61(7):70311, jul 2018. ISSN 1674-7348. DOI: 10.1007/s11433-0179181-9.

[15] Markus Müller, Sebastian Diehl, Guido Pupillo, and Peter Zoller. Engineered Open Systems and Quantum Simulations with Atoms and Ions. In Paul Berman, Ennio Arimondo, and Chun Lin, editors, Adv. Atom. Mol. Opt. Phy. 61, pages 1-80. Academic Press, 2012. ISBN 0123964822. DOI: 10.1016/B978-0-12-396482-3.00001-6.

[16] Chao Shen, Kyungjoo Noh, Victor V. Albert, Stefan Krastanov, Michel H. Devoret, Robert J. Schoelkopf, S. M. Girvin, and Liang Jiang. Quantum channel construction with circuit quantum electrodynamics. Phys. Rev. B, 95(13):134501, apr 2017. ISSN 2469-9950. DOI: 10.1103/PhysRevB.95.134501.

[17] Francesco Ticozzi and Lorenza Viola. Quantum and classical resources for unitary design of open-system evolutions. Quantum Sci. Technol., 2(3):034001, sep 2017. ISSN 2058-9565. DOI: $10.1088 / 2058-9565 /$ aa722a.

[18] A. A. Belavin, B. Ya. Zel'dovich, A. M. Perelomov, and V. S. Popov. Relaxation of Quantum Systems with Equidistant
Spectra. Sov. Phys. JETP-USSR, 29(1): 145, 1969. URL http://www.jetp.ac.ru/ cgi-bin/e/index/e/29/1/p145?a=list.

[19] G. Lindblad. On the generators of quantum dynamical semigroups. Commun. Math. Phys., 48 (2):119-130, jun 1976. ISSN 0010-3616. DOI: $10.1007 /$ BF01608499.

[20] V. Gorini, A. Kossakowski, and E. C. G. Sudarshan. Completely positive dynamical semigroups of N-level systems. J. Math. Phys., 17:821, may 1976. ISSN 00222488. DOI: $10.1063 / 1.522979$.

[21] Thomas Banks, Leonard Susskind, and Michael E. Peskin. Difficulties for the evolution of pure states into mixed states. Nucl. Phys. B, 244:125-134, sep 1984. ISSN 05503213. DOI: 10.1016/0550-3213(84)90184-6.

[22] Michael M. Wolf and David Perez-Garcia. The inverse eigenvalue problem for quantum channels. 2010. URL http://arxiv.org/abs/ 1005.4545 .

[23] Michael M. Wolf. Quantum Channels \& Operations Guided Tour, 2010. URL http: //www-m5. ma.tum.de/foswiki/pub/M5/Allgemeines/ MichaelWolf/QChannelLecture.pdf.

[24] Robin Blume-Kohout, Hui Khoon Ng, David Poulin, and Lorenza Viola. Informationpreserving structures: A general framework for quantum zero-error information. Phys. Rev. A, 82(6):062306, dec 2010. ISSN 1050-2947. DOI: 10.1103/PhysRevA.82.062306.

[25] Ji Guan, Yuan Feng, and Mingsheng Ying. The Structure of Decoherence-free Subsystems. feb 2018. URL http://arxiv.org/abs/1802. 04904.

[26] J. Novotny, G. Alber, and I. Jex. Asymptotic properties of quantum Markov chains. J. Phys. A Math. Theor., 45(48):485301, dec 2012. ISSN 1751-8113. DOI: 10.1088/1751$8113 / 45 / 48 / 485301$.

[27] D Burgarth, G Chiribella, V Giovannetti, $\mathrm{P}$ Perinotti, and $\mathrm{K}$ Yuasa. Ergodic and mixing quantum channels in finite dimensions. New J. Phys., 15(7):073045, jul 2013. ISSN 1367-2630. DOI: $10.1088 / 1367-2630 / 15 / 7 / 073045$.

[28] J. Novotný, J. Maryška, and Igor Jex. Quantum Markov processes: From attractor structure to explicit forms of asymptotic states. Eur. Phys. J. Plus, 133(8):310, aug 2018. ISSN 2190-5444. DOI: $10.1140 /$ epjp/i2018-12109-8.

[29] Ulrich Groh. The peripheral point spectrum of schwarz operators onC *-algebras. Math. Zeitschrift, 176(3):311-318, sep 1981. ISSN 0025-5874. DOI: 10.1007/BF01214608.

[30] Bei Zeng, Xie Chen, Duan-Lu Zhou, and XiaoGang Wen. Quantum Information Meets Quantum Matter. Quantum Science and Technology. Springer New York, New York, NY, aug 2019. 
ISBN 978-1-4939-9082-5. DOI: 10.1007/978-14939-9084-9.

[31] Sven Bachmann and Bruno Nachtergaele. Product vacua with boundary states. Phys. Rev. B, 86(3):035149, jul 2012. ISSN 1098-0121. DOI: 10.1103/PhysRevB.86.035149.

[32] Sven Bachmann and Bruno Nachtergaele. Product Vacua with Boundary States and the Classification of Gapped Phases. Commun. Math. Phys., 329(2):509-544, jul 2014. ISSN 00103616. DOI: $10.1007 / \mathrm{s} 00220-014-2025-\mathrm{x}$.

[33] D. Pérez-García, M. M. Wolf, M. Sanz, F. Verstraete, and J. I. Cirac. String Order and Symmetries in Quantum Spin Lattices. Phys. Rev. Lett., 100(16):167202, apr 2008. ISSN 00319007. DOI: 10.1103/PhysRevLett.100.167202.

[34] Norbert Schuch, Ignacio Cirac, and David Pérez-García. PEPS as ground states: Degeneracy and topology. Ann. Phys. (N. Y)., 325 (10):2153-2192, oct 2010. ISSN 00034916. DOI: 10.1016/j.aop.2010.05.008.

[35] Nick Bultinck, Dominic J. Williamson, Jutho Haegeman, and Frank Verstraete. Fermionic matrix product states and one-dimensional topological phases. Phys. Rev. B, 95(7):075108, feb 2017. ISSN 2469-9950. DOI: 10.1103/PhysRevB.95.075108.

[36] Jutho Haegeman, Michaël Mariën, Tobias J. Osborne, and Frank Verstraete. Geometry of matrix product states: Metric, parallel transport, and curvature. J. Math. Phys., 55(2): 021902, feb 2014. ISSN 0022-2488. DOI: $10.1063 / 1.4862851$.

[37] Gemma De las Cuevas, J Ignacio Cirac, Norbert Schuch, and David Perez-Garcia. Irreducible forms of matrix product states: Theory and applications. J. Math. Phys., 58(12):121901, dec 2017. ISSN 0022-2488. DOI: 10.1063/1.5000784.

[38] Laurens Vanderstraeten, Jutho Haegeman, and Frank Verstraete. Tangent-space methods for uniform matrix product states. SciPost Phys. Lect. Notes, page 7, jan 2019. ISSN 2590-1990. DOI: 10.21468/SciPostPhysLectNotes.7.

[39] E. C. G. Sudarshan, P. M. Mathews, and Jayaseetha Rau. Stochastic Dynamics of Quantum-Mechanical Systems. Phys. Rev., 121 (3):920-924, feb 1961. ISSN 0031-899X. DOI: 10.1103/PhysRev.121.920.

[40] K Kraus. General state changes in quantum theory. Ann. Phys., 64(2):311-335, jun 1971. ISSN 00034916. DOI: 10.1016/0003-4916(71)90108-4.

[41] Man-Duen Choi. Completely positive linear maps on complex matrices. Linear Algebra Appl., 10(3):285-290, jun 1975. ISSN 00243795. DOI: 10.1016/0024-3795(75)90075-0.

[42] Raffaella Carbone and Anna Jenčová. On period, cycles and fixed points of a quantum chan- nel. may 2019. URL http://arxiv.org/abs/ 1905.00857.

[43] Carlton M. Caves. Quantum Error Correction and Reversible Operations. J. Supercond., 12(6):707-718, 1999. ISSN 08961107. DOI: 10.1023/A:1007720606911.

[44] Göran Lindblad. A General No-Cloning Theorem. Lett. Math. Phys., 47(2): 189-196, 1999. ISSN 03779017. DOI: 10.1023/A:1007581027660.

[45] Robin Blume-Kohout, Hui Khoon Ng, David Poulin, and Lorenza Viola. Characterizing the structure of preserved information in quantum processes. Phys. Rev. Lett., 100(3):030501, jan 2008. ISSN 0031-9007. DOI: 10.1103/PhysRevLett.100.030501.

[46] Bernhard Baumgartner and Heide Narnhofer. The Structures of State Space Concerning Quantum Dynamical Semigroups. Rev. Math. Phys., 24:1250001, mar 2012. ISSN 0129-055X. DOI: $10.1142 /$ S0129055X12500018.

[47] Raffaella Carbone and Yan Pautrat. Irreducible Decompositions and Stationary States of Quantum Channels. Rep. Math. Phys., 77(3):293313, 2016. ISSN 00344877. DOI: 10.1016/S00344877(16)30032-5.

[48] David E. Evans and Raphael Hoegh-Krohn. Spectral Properties of Positive Maps on C*Algebras. J. London Math. Soc., s2-17(2): 345-355, apr 1978. ISSN 00246107. DOI: $10.1112 / \mathrm{jlms} / \mathrm{s} 2-17.2 .345$.

[49] B. V. Rajarama Bhat, Robin Hillier, Nirupama Mallick, and Vijaya Kumar U. Roots of Completely Positive Maps. dec 2018. URL http: //arxiv.org/abs/1812.08123.

[50] Karl Alicki and Robert Lendi. Quantum Dynamical Semigroups and Applications, volume 717 of Lecture Notes in Physics. Springer Berlin Heidelberg, Berlin, Heidelberg, 2007. ISBN 9783-540-70860-5. DOI: 10.1007/3-540-70861-8.

[51] S. G. Schirmer and Xiaoting Wang. Stabilizing open quantum systems by Markovian reservoir engineering. Phys. Rev. A, 81:062306, jun 2010. DOI: 10.1103/PhysRevA.81.062306.

[52] Matteo Ippoliti, Leonardo Mazza, Matteo Rizzi, and Vittorio Giovannetti. Perturbative approach to continuous-time quantum error correction. Phys. Rev. A, 91(4):042322, apr 2015. ISSN 1050-2947. DOI: 10.1103/PhysRevA.91.042322.

[53] V. I. Oseledets. Completely positive linear mappings, non-Hamiltonian evolution, and quantum stochastic processes. J. Sov. Math., 25(6): 1529-1557, jun 1984. ISSN 0090-4104. DOI: 10.1007/BF01101650.

[54] Maxim Raginsky. Strictly contractive quantum channels and physically realizable quantum computers. Phys. Rev. A, 65(3):032306, 
feb 2002. ISSN 1050-2947. DOI: 10.1103/PhysRevA.65.032306.

[55] Maxim Raginsky. Dynamical Aspects of Information Storage in Quantum-Mechanical Systems. $\mathrm{PhD}$ thesis, Northwestern University, jul 2002. URL http://arxiv.org/abs/ quant-ph/0207162.

[56] Daniel Burgarth and Vittorio Giovannetti. The generalized Lyapunov theorem and its application to quantum channels. New J. Phys., 9 (5):150-150, may 2007. ISSN 1367-2630. DOI: 10.1088/1367-2630/9/5/150.

[57] E. B. Davies. Quantum stochastic processes II. Commun. Math. Phys., 19(2):83-105, jun 1970. ISSN 0010-3616. DOI: 10.1007/BF01646628.

[58] Mikel Sanz, David Perez-Garcia, Michael M. Wolf, and Juan I. Cirac. A Quantum Version of Wielandt's Inequality. IEEE Trans. Inf. Theory, 56(9):4668-4673, sep 2010. ISSN 00189448. DOI: 10.1109/TIT.2010.2054552.

[59] Victor V. Albert, Barry Bradlyn, Martin Fraas, and Liang Jiang. Geometry and Response of Lindbladians. Phys. Rev. X, 6(4):041031, nov 2016. ISSN 2160-3308. DOI: 10.1103/PhysRevX.6.041031.

[60] Ji Guan, Yuan Feng, and Mingsheng Ying. Decomposition of quantum Markov chains and its applications. J. Comput. Syst. Sci., 95: 55-68, aug 2018. ISSN 00220000. DOI: 10.1016/j.jcss.2018.01.005.

[61] David W. Kribs. Quantum channels, wavelets, dilations and representations of On. Proc. Edinburgh Math. Soc., 46(2): S0013091501000980, jun 2003. ISSN 00130915. DOI: 10.1017 /S0013091501000980.

[62] Man-Duen Choi and David W. Kribs. Method to Find Quantum Noiseless Subsystems. Phys. Rev. Lett., 96(5):050501, feb 2006. ISSN 00319007. DOI: 10.1103/PhysRevLett.96.050501.

[63] Aurelian Gheondea. On Propagation of Fixed Points of Quantum Operations and Beyond. nov 2016. URL http://arxiv . org/abs/1611. 04742.

[64] Aurelian Gheondea. Symmetries Versus Conservation Laws in Dynamical Quantum Systems: A Unifying Approach Through Propagation of Fixed Points. Ann. Henri Poincare, 19 (6):1787-1816, jun 2018. ISSN 1424-0637. DOI: 10.1007/s00023-018-0666-6.

[65] Oleg Szehr and Michael M. Wolf. Connected components of irreducible maps and 1D quantum phases. J. Math. Phys., 57(8):081901, aug 2016. ISSN 0022-2488. DOI: 10.1063/1.4960557.

[66] Sven Bachmann, Spyridon Michalakis, Bruno Nachtergaele, and Robert Sims. Automorphic Equivalence within Gapped Phases of Quantum Lattice Systems. Commun. Math. Phys., 309
(3):835-871, feb 2012. ISSN 0010-3616. DOI: 10.1007/s00220-011-1380-0.

[67] John E. Gough, Tudor S. Ratiu, and Oleg G. Smolyanov. Noether's theorem for dissipative quantum dynamical semi-groups. J. Math. Phys., 56(2):022108, feb 2015. ISSN 0022-2488. DOI: $10.1063 / 1.4907985$.

[68] Victor V. Albert. Lindbladians with multiple steady states: theory and applications. $\mathrm{PhD}$ thesis, Yale University, 2017. URL https: //arxiv.org/abs/1802.00010.

[69] Iman Marvian and Robert W Spekkens. Extending Noether's theorem by quantifying the asymmetry of quantum states. Nat. Commun., 5(1):3821, sep 2014. ISSN 2041-1723. DOI: $10.1038 /$ ncomms4821.

[70] Shenggang Ying, Yuan Feng, Nengkun Yu, and Mingsheng Ying. Reachability Probabilities of Quantum Markov Chains. In Pedro R. D'Argenio and Hernán Melgratti, editors, $C O N$ CUR 2013 - Concurr. Theory, pages 334348. Springer Berlin Heidelberg, 2013. DOI: 10.1007/978-3-642-40184-8_24.

[71] Giuseppe Ilario Cirillo and Francesco Ticozzi. Decompositions of Hilbert spaces, stability analysis and convergence probabilities for discrete-time quantum dynamical semigroups. J. Phys. A Math. Theor., 48(8):085302, feb 2015. ISSN 1751-8113. DOI: $10.1088 / 1751-$ $8113 / 48 / 8 / 085302$.

[72] J. G. Kemeny and J. L. Snell. Finite Markov chains. Springer-Verlag, New York, 2nd edition, 1983. URL https://www.springer.com/ us/book/9780387901923.

[73] Daniel A. Lidar, Isaac L. Chuang, and K. Birgitta Whaley. Decoherence-Free Subspaces for Quantum Computation. Phys. Rev. Lett., 81:2594, sep 1998. ISSN 0031-9007. DOI: 10.1103/PhysRevLett.81.2594.

[74] Daniel A. Lidar and K. Birgitta Whaley. Decoherence-Free Subspaces and Subsystems. In Fabio Benatti and Roberto Floreanini, editors, Irreversible Quantum Dyn., volume 622 of Lecture Notes in Physics, chapter 5, page 83. Springer, Berlin, Heidelberg, jun 2003. ISBN 978-3-540-40223-7. DOI: 10.1007/3-540-448748.

[75] Raisa I. Karasik, Karl-Peter Marzlin, Barry C. Sanders, and K. Birgitta Whaley. Criteria for dynamically stable decoherence-free subspaces and incoherently generated coherences. Phys. Rev. A, 77(5):052301, may 2008. ISSN 10502947. DOI: 10.1103/PhysRevA.77.052301.

[76] Takeo Kamizawa. Algebraic Method in the Analysis of Decoherence-Free Subspaces in Open Quantum Systems. Int. J. Theor. Phys., pages 1-13, jan 2018. DOI: 10.1007/s10773-0173657-3. 
[77] Emanuel Knill, Raymond Laflamme, and Lorenza Viola. Theory of Quantum Error Correction for General Noise. Phys. Rev. Lett., 84: 2525-2528, mar 2000. ISSN 0031-9007. DOI: 10.1103/PhysRevLett.84.2525.

[78] Mizanur Rahaman. Multiplicative properties of quantum channels. J. Phys. A Math. Theor., 50 (34):345302, aug 2017. ISSN 1751-8113. DOI: 10.1088/1751-8121/aa7b57.

[79] John A. Holbrook, David W. Kribs, and Raymond Laflamme. Noiseless Subsystems and the Structure of the Commutant in Quantum Error Correction. Quantum Inf. Process., 2(5): 381-419, oct 2003. ISSN 1570-0755. DOI: 10.1023/B:QINP.0000022737.53723.b4.

[80] E. Knill. Protected realizations of quantum information. Phys. Rev. A, 74(4):042301, oct 2006. ISSN 1050-2947. DOI: 10.1103/PhysRevA.74.042301.

[81] Takanori Maehara and Kazuo Murota. A numerical algorithm for block-diagonal decomposition of matrix *-algebras with general irreducible components. Jpn. J. Ind. Appl. Math., 27(2):263-293, sep 2010. ISSN 0916-7005. DOI: $10.1007 / \mathrm{s} 13160-010-0007-8$.

[82] Xiaoting Wang, Mark Byrd, and Kurt Jacobs. Numerical method for finding decoherence-free subspaces and its applications. Phys. Rev. A, 87(1):012338, jan 2013. ISSN 1050-2947. DOI: 10.1103/PhysRevA.87.012338.

[83] Ion Nechita and Clément Pellegrini. Random repeated quantum interactions and random invariant states. Probab. Theory Relat. Fields, 152 (1-2):299-320, feb 2012. ISSN 0178-8051. DOI: 10.1007/s00440-010-0323-6.

[84] Laurent Bruneau, Alain Joye, and Marco Merkli. Repeated interactions in open quantum systems. J. Math. Phys., 55(7):075204, jul 2014. ISSN 0022-2488. DOI: 10.1063/1.4879240.

[85] M. Born and V. Fock. Beweis des Adiabatensatzes. Zeitschrift Phys., 51(3-4):165180, mar 1928. ISSN 1434-6001. DOI: 10.1007/BF01343193.

[86] Tosio Kato. On the adiabatic theorem of quantum mechanics. J. Phys. Soc. Jpn., 5:435, 1950. DOI: $10.1143 /$ JPSJ.5.435.

[87] M. V. Berry. Quantal Phase Factors Accompanying Adiabatic Changes. Proc. R. Soc. A, 392: 45-57, mar 1984. DOI: 10.1098/rspa.1984.0023.

[88] Frank Wilczek and A. Zee. Appearance of Gauge Structure in Simple Dynamical Systems. Phys. Rev. Lett., 52(24):2111, jun 1984. ISSN 0031-9007. DOI: 10.1103/PhysRevLett.52.2111.

[89] J. E. Avron, M. Fraas, G. M. Graf, and P. Grech. Adiabatic Theorems for Generators of Contracting Evolutions. Commun. Math. Phys., 314(1): 163-191, may 2012. ISSN 0010-3616. DOI: 10.1007/s00220-012-1504-1.
[90] G Nenciu and G Rasche. On the adiabatic theorem for nonself-adjoint Hamiltonians. $J$. Phys. A Math. Gen., 25(21):5741-5751, nov 1992. ISSN 0305-4470. DOI: 10.1088/03054470/25/21/027.

[91] M V Berry and R Uzdin. Slow non-Hermitian cycling: exact solutions and the Stokes phenomenon. J. Phys. A Math. Theor., 44(43): 435303, oct 2011. ISSN 1751-8113. DOI: 10.1088/1751-8113/44/43/435303.

[92] Raam Uzdin, Alexei Mailybaev, and Nimrod Moiseyev. On the observability and asymmetry of adiabatic state flips generated by exceptional points. J. Phys. A Math. Theor., 44(43):435302, oct 2011. ISSN 1751-8113. DOI: 10.1088/1751$8113 / 44 / 43 / 435302$.

[93] J. Holler, N. Read, and J.G.E. Harris. NonHermitian adiabatic transport in the space of exceptional points. 2019. URL http://arxiv . org/abs/1809.07175.

[94] Ronald L. Graham, Donald E. Knuth, and Oren Patashnik. Concrete Mathematics: a Foundation for Computer Science. Addison-Wesley, New York, 2nd edition, 1994. URL https://www.worldcat.org/title/ concrete-mathematics-a-foundation-for-computer-sc oclc/29357079.

[95] M. S. Sarandy and Daniel A. Lidar. Abelian and non-Abelian geometric phases in adiabatic open quantum systems. Phys. Rev. A, 73(6):062101, jun 2006. ISSN 1050-2947. DOI: 10.1103/PhysRevA.73.062101.

[96] Barry Simon. Holonomy, the Quantum Adiabatic Theorem, and Berry's Phase. Phys. Rev. Lett., 51(24):2167, dec 1983. ISSN 0031-9007. DOI: 10.1103/PhysRevLett.51.2167.

[97] Lorenzo Campos Venuti, Tameem Albash, Daniel A. Lidar, and Paolo Zanardi. Adiabaticity in open quantum systems. Phys. Rev. A, 93 (3):032118, mar 2016. ISSN 2469-9926. DOI: 10.1103/PhysRevA.93.032118.

[98] Hiroshi Ueda, Isao Maruyama, and Kouichi Okunishi. Uniform Matrix Product State in the Thermodynamic Limit. J. Phys. Soc. Jpn., 80 (2):023001, feb 2011. ISSN 0031-9015. DOI: 10.1143 /JPSJ.80.023001.

[99] F. Verstraete, J. I. Cirac, J. I. Latorre, E. Rico, and M. M. Wolf. Renormalization-Group Transformations on Quantum States. Phys. Rev. Lett., 94(14):140601, apr 2005. ISSN 0031-9007. DOI: 10.1103/PhysRevLett.94.140601.

[100] Tzu-Chieh Wei. Entanglement under the renormalization-group transformations on quantum states and in quantum phase transitions. Phys. Rev. A, 81(6):062313, jun 2010. ISSN 1050-2947. DOI: 10.1103/PhysRevA.81.062313.

[101] J. I. Cirac, D. Pérez-García, N. Schuch, and 
F. Verstraete. Matrix product density operators: Renormalization fixed points and boundary theories. Ann. Phys., 378:100149, mar 2017. ISSN 00034916. DOI: 10.1016/j.aop.2016.12.030.

[102] J. Ignacio Cirac, Didier Poilblanc, Norbert Schuch, and Frank Verstraete. Entanglement spectrum and boundary theories with projected entangled-pair states. Phys. Rev. B, 83(24):245134, 2011. ISSN 1098-0121. DOI: 10.1103/PhysRevB.83.245134.

[103] Michal Bialonczyk, Andrzej Jamiolkowski, and Karol Zyczkowski. Application of Shemesh theorem to quantum channels. J. Math. Phys., 59 (10):102204, oct 2018. ISSN 0022-2488. DOI: $10.1063 / 1.5027616$. 